Cómo citar este trabajo: Hernández Luis, J. Á. (2021). Inter-island air accessibility and territorial cohesion in the Canary Islands. Boletín de la Asociación de Geógrafos Españoles, (89). https://doi.org/10.21138/bage.3112

\title{
Accesibilidad aérea interinsular y cohesión territorial en Canarias
}

Inter-island air accessibility and territorial cohesion in the Canary Islands

\author{
José Ángel Hernández Luis \\ jose.hernandez@ulpgc.es \\ Departmento de Geografía \\ Universidad de Las Palmas de Gran Canaria (España)
}

\section{Resumen}

Este trabajo tiene como objetivo realizar un análisis de la actual accesibilidad puerta - puerta del transporte aéreo interinsular como elemento de cohesión territorial de Canarias. Desde el punto de vista metodológico, se realiza un diagnóstico considerando varios aspectos claves: frecuencias, disponibilidad de asientos, tarifas puerta - puerta y su impacto en los perfiles de viajeros de mayor demanda, además de los horarios que, conjuntamente con los tiempos de transporte, nos proporcionan en los destinos una disponibilidad horaria final para el viajero, todo ello en un viaje de ida y vuelta y en una misma jornada. La conclusión es que la accesibilidad ha mejorado sustancialmente en los últimos años, pero existen marcadas carencias de disponibilidad de tiempo en los destinos que imponen elevados sobrecostes al viajero, aspecto al que hasta ahora se le ha prestado escasa atención, pues los estudios se han centrado, sobre todo, en las tarifas aéreas. En suma, ello conlleva un déficit de integración territorial, especialmente para los residentes en las islas de El Hierro y de La Gomera y que debe ser enmendado tanto por la administración, ampliando los horarios de operatividad de las infraestructuras aeroportuarias, como por las compañías aéreas, procediendo de igual manera con sus horarios. 
Palabras clave: accesibilidad aérea; cohesión territorial; disponibilidad de tiempo; obligación de servicio público.

\begin{abstract}
This paper makes a study of the current door-to-door accessibility of inter-island air transport as an element of territorial cohesion in the Canary Islands. The methodology used makes a diagnosis considering several key aspects: frequencies, seat availability, door-to-door rates and their impact on the profiles of travellers with the highest demand. In the same way, the schedules that, together with the transport times, provide us in the destinations with a final hourly availability for the traveller, all in a round trip and in the same day. We conclude that accessibility has improved considerably in recent years, but there is a lack of time availability for travellers in some destinations, imposing high extra costs for them, an aspect to which little attention has been given, since the studies have only focused mainly on airfares. In summary, there is a territorial integration deficit, especially for residents of the islands of El Hierro and La Gomera, which must be corrected by the administration, extending the operating hours of the airport infrastructures, and also by the airlines, also expanding their hours of operation.
\end{abstract}

Key words: air accessibility; territorial cohesion; time availability; public service obligation.

\title{
1 Introducción
}

El estudio se centra en analizar la accesibilidad aérea interinsular en Canarias, partiendo de variables como las frecuencias, tarifas, horarios, disponibilidad de asientos, etc. No obstante, haremos hincapié en los horarios de operatividad, en tanto que generan una determinada disponibilidad de tiempo en destino en un sistema round trip con todas sus consecuencias. Del diagnóstico realizado, se deriva que son estos últimos uno de los principales hándicaps en aras de avanzar hacia una efectiva integración territorial del archipiélago y, para mayor rigor del análisis, esta accesibilidad horaria se calcula en un sistema puerta - puerta, como marcan las políticas de transporte más relevantes de la Unión Europea (European Commission, 2001, 2011).

Partimos de la base de que el transporte interinsular es sumamente importante para las islas y, en particular, para Canarias. Este último es muy utilizado por motivos laborales, comerciales, de negocios, sanitarios, administrativos, etc., siendo por tanto muy elevados los viajes entre islas que se realizan dentro de una misma jornada. Por ello mismo, el transporte interinsular -aéreo y marítimo-, es muy susceptible a cambios de horarios, disponibilidad de plazas en determinados 
días y horas, un mínimo de tiempo disponible en destino, etc. Son todos estos factores los que nos muestran unos determinados índices de calidad y que repercuten directamente en la socioeconomía de las Islas. Estos índices, entre otros, redundan en la filosofía de la Red Transeuropea de Transportes, es decir, el de la integración de los ciudadanos de la Unión Europea, donde la accesibilidad es clave (Coccossis, 1995; European Commission, 2011).

En el trabajo realizamos un breve esbozo del marco territorial de Canarias, para a continuación presentar el transporte aéreo en las Islas como una herramienta fundamental para el desarrollo del archipiélago. Posteriormente, un análisis de accesibilidad básico (frecuencias, tarifas, etc.), desembocará en uno de los objetivos principales del trabajo como es el estudio de los horarios que da lugar a una determinada disponibilidad de tiempo del viajero. Lejos de considerar el tiempo de viaje como algo útil para el descanso o el trabajo de oficina trasladado al asiento de un medio de transporte (Jain \& Lyons, 2008), la llegada al destino, particularmente en un horario temprano, es absolutamente vital para la cohesión territorial (Murillo Fort, 1992; Cross \& Nutley, 1999; Neutens, 2012). Para el cálculo de esta accesibilidad temporal, desarrollamos una metodología entre capitales insulares y, por tanto, basada en el sistema puerta - puerta, tomándose como ejemplo las programaciones invernales de los diversos operadores aéreos entre 2000 y 2020.

\section{Revisión de la literatura}

El transporte ha adquirido en las sociedades actuales una relevancia cada vez mayor, sobre todo por las políticas de especialización del territorio y la globalización (Hoyle \& Knowles, 1998; Rodrigue, 2006). En efecto, si la literatura es abundante, exponiendo la dependencia entre transporte y desarrollo - sea este sostenible o no-, para el caso de las islas esta relación es condición sine qua non (Hoyle, 1999), donde el autor expone que sin transporte y, además, que este presente unas mínimas condiciones de accesibilidad física, económica y de tiempo, la competitividad de estos territorios se ve amenazada. Por tanto, la accesibilidad exterior de las islas presenta una clara desventaja en comparación con los territorios continentales, debido a su evidente discontinuidad territorial, acompañada de la lejanía, que genera mayores costes, mayor tiempo de transporte marítimo y/o aéreo, etc. A ello hay que unirle, por lo general, la ausencia total de materias primas que las hacen depender aún más de los sistemas de transporte para su importación, por lo que la dependencia de otros territorios es extrema. Todo ello se ha recogido, como decíamos, en una extensa bibliografía, destacando en especial los siguientes trabajos en esta línea: (Brookfield, 1980; Gobierno de Canarias, 1998; Bowen, 2000; Ramos 
Pérez, 2001; Tsekeris, 2009; Gobierno de Canarias, 2010; Minato \& Morimoto, 2011; Deidda, 2016; UTE Eco-CoRe, 2019).

Una parte significativa de los trabajos que abordan la problemática insular, se han centrado en evaluar los costes diferenciales que supone vivir - o desarrollar cualquier actividad económica-, en las islas frente al continente, centrándose en los costes económicos y de tiempo, aunque este último también se traduce en un impacto económico. Así pues, clásico e inevitable citar, es el estudio inédito del Ministerio de Transportes, Turismo y Comunicaciones, que afirmaba el sobrecoste de los productos comercializados en Canarias y que, genéricamente, lo cifraba entre un 8 y hasta casi un 20 \% en algunas mercaderías, en relación con la Península (MTTC, 1981). Pero sin duda, el punto de inflexión para comprender en su esencia el problema de la insularidad en Canarias, va a ser el trabajo de Murillo Fort, donde instrumentalizó la diferencia de costes del transporte entre Canarias y el continente en aras de proveer, entre otros objetivos, de mecanismos para la asignación de los Fondos de Compensación Interterritorial (Murillo Fort, 1992). En este sentido, el trabajo de Murillo profundizó en aspectos muy sensibles para los habitantes y economía de las Islas, desagregando incluso los datos a nivel insular y poniendo de manifiesto el agravamiento del inconveniente del transporte en las islas no capitalinas (problemas de horarios, frecuencias, falta de cupo para las mercancías o ausencia de plazas aéreas, etc.). Escasos años antes, Bardolet, aunque para Baleares, hacía hincapié en dicha condición diferencial con el continente, indicando que el "transporte es vital para un desarrollo aceptable de riqueza y de bienestar social", aunque apostillando también que el desarrollo de este podía convertirse en "el fin de la singular condición de espacio protegido" (Bardolet, 1989, p. 11) que conlleva el aislamiento territorial, más preocupado por el impacto del Medio y sus consecuencias sobre la imagen turística. Por su parte, en el Libro Blanco de los Transportes en Canarias, se ponía énfasis en el hecho diferencial y sus costes, incluso entre islas, realizando un "índice de dependencia insular" que relegaba a las islas de El Hierro y de La Gomera a una dependencia máxima, mientras que Tenerife y Gran Canaria oscilaban en valores entre el 20 y el 30 \% de la citada dependencia, en este último caso con respecto a la Península (Gobierno de Canarias, 1998). Otros autores, para el caso de las casi trescientas islas de la Unión Europea, exponían esta disparidad de desarrollo, aludiendo a que el PIB per cápita de estos territorios solo representaba el 72 \% de la media de la Unión, apuntando al concepto de los costes negativos de la insularidad (Manera \& Garau, 2005). También las islas italianas, a pesar de su cercanía al continente y mayor extensión superficial, centran su debilidad y fragilidad económica en el transporte, escenificando sobre todo el sobrecoste en la cesta de la compra, además de las 
dificultades para potenciar cualquier actividad económica (Paci, 1997; Butera, 2002). En Azores, otros autores perciben un mayor problema en las infraestructuras de transporte y cómo estas pueden canalizar un desarrollo sostenible de la conectividad interinsular, fomentando la economía (Castanho, 2021). En las Antillas francesas, algunos autores centran la problemática del transporte con la metrópoli en una cuestión política, sobre todo en materia de desregulación, fomento del turismo, etc. (Ranély Vergé-Dépré, 2005). Por su parte, el Gobierno de Canarias cifraba en 2001 en más de 3.500 millones de euros anuales el coste de la ultraperiferia, siendo de 82 millones el sobrecoste imputable a los viajes de negocios. Estos valores, en términos porcentuales, eran más elevados en las islas no capitalinas y empresas de escasa fuerza de trabajo y capitalización (Fundación Tomillo, 2001). Otro estudio, con datos de 2016, elevaba el coste de la ultraperiferia a 5228 millones de euros anuales (el 8\% de la facturación de las empresas), de los que 326 millones (el 0,5\% de la facturación), eran imputables a los viajes de negocios, es decir, casi un 300 \% más en relación con el año 2001, corroborando a su vez el sobrecoste aún mayor de las islas no capitalinas (UTE Eco-CoRe, 2019). En suma, parece desprenderse de todos estos análisis un problema de debilidad y falta de oportunidad, tanto para los viajeros como para las mercancías y cuyo pilar, en gran medida, está condicionado por una accesibilidad que siempre será más endeble en las islas frente al continente, pero donde la búsqueda de alternativas para minimizar el impacto de la lejanía y la fragmentación territorial debe ser el centro del debate.

Como decimos, uno de los grandes problemas de las islas es la debilidad de su accesibilidad, en tanto que muestra la capacidad de moverse en el territorio y, además, con implicaciones de empleo de tiempo significativo (Pooler, 1995; O'Sullivan, 2000). Así pues, la accesibilidad física, es decir, la existencia o no de ruta directa y cuántas frecuencias existen en un determinado período; la económica, analizada por ejemplo a través de las tarifas de un operador de transporte; o la temporal, medida en unidades de tiempo entre un nodo y otro de la red, se constituyen en los pilares básicos de la evaluación potencial que la accesibilidad puede transferir a una sociedad para su desarrollo socioeconómico (Mavoa, 2012; Antunes, 2018). Coincidimos además con muchos autores e informes cuando argumentan que el mejor análisis de la accesibilidad en los diferentes medios de transporte es aquél que estudia el sistema puerta - puerta (European Commission 2011; Durán-Hormazábal, 2016; Yatskiv, 2017; Tahmasbia, 2019), pues nos aproxima a los verdaderos costes y tiempos de viaje y, por ende, nos acerca a la evaluación de la cohesión territorial que ya intentaba potenciar el Gobierno de 
Canarias, mediados los años ochenta y comienzos de los noventa, a través de la creación de una empresa de tercer nivel aéreo (Metra/Seis, 1985; Sri International, 1992).

Bien es verdad que la cohesión territorial puede ser tomada desde diferentes perspectivas: física, social, justicia espacial, etc. (Fernández Tabales, 2009), pero en este trabajo se abordará la imputable al grado de integración que se deriva del transporte, siguiendo las líneas de políticas de gran impacto en los últimos años como la Red Transeuropea de Transportes (Commission of the European Communities, 2005), o donde preferentemente se estudian las redes de transporte en medios insulares en aras de la mentada integración (Hernández Luis, 2004; Antunes, 2018; Martín Osante, 2020; Ramos Pérez, 2020).

Hasta los años setenta la relación entre el espacio y el tiempo no había sido abordada en profundidad en los trabajos de geografía. En este sentido, la escuela sueca de Lund se puede considerar una de las pioneras desde este punto de vista, tomando un análisis donde el espacio y el tiempo para la población se mostraban de manera dialéctica en una jornada cotidiana (Hägerstrand, 1970). En realidad, su aportación estaba realizando un análisis de accesibilidad territorial y, en especial, partiendo del tiempo empleado en el transporte y que luego sería desarrollado por otros autores (Thrift \& Pred, 1981; Ashiru, 2003; Harris, 2004; Ettema, 2007; Soo, 2009; Kim, 2013). Con el paso de los años, uno de los principales objetivos del concepto de la accesibilidad, que ya intuía Hägerstrand, ha tenido como objeto alcanzar los destinos a costos y tiempos razonables, hasta el punto de que el valor del tiempo en la economía del transporte, se ha convertido en uno de los pilares fundamentales de los análisis de la accesibilidad (Gwilliam, 1997; Hernández Luis, 2002; Liu \& Zhu, 2004; Yamaguchi, 2007; Jain \& Lions, 2008; Chang, 2010; Lei \& la Iglesia, 2010; Benenson, 2011; Grubesic \& Fangwu, 2013; Price \& Matthews, 2013; Chowdhury, 2015). Y es que, según algunos autores, el valor del tiempo es el costo de oportunidad que cada persona utiliza a lo largo de su vida; en el caso particular del tiempo destinado a transportarse, es la disposición que cada persona tiene que sufragar por reducir sus tiempos de viaje o la compensación que está dispuesta a recibir por perder tiempo (Wardman, 1998). En la sociedad actual la falta de tiempo se ha convertido en un importante problema social que se aprecia en prácticamente todos los ámbitos de la sociedad (Glorieux, 2008). A su vez, los efectos negativos de la escasez de tiempo, además de sus implicaciones económicas, perturban el bienestar de las personas, pudiendo producir un desequilibrio entre el trabajo y la vida, una menor satisfacción familiar, problemas como el estrés, además de fatiga y agotamiento entre otros (Pelfrene, 2001; Ritsema van Eck, 2005). No es casualidad por tanto que, en la actualidad, exista un creciente interés por relacionar los 
tiempos de desplazamiento cortos y la calidad de vida, como se desprende del surgimiento del concepto de las ciudades de los 15 minutos (Li, 2019).

Pues bien, hace ya más de dos décadas y dada la relevancia del factor tiempo para los viajes interinsulares, el Libro Blanco de los Transportes en Canarias, hizo hincapié en la importancia de la accesibilidad interinsular entre las Islas mostrando, a través de la cartografía, la alta desigualdad existente a través de las isócronas y los isocostes y en comparación con la Península Ibérica (Gobierno de Canarias, 1998). Para ello se tomaban sobre todo los transportes marítimos y aéreos como complementarios y nunca como competidores entre sí (Ray, 1985; Rigas, 2009; Hernández Luis, 2018).

De modo más específico, algunos trabajos se han detenido en analizar la incidencia que ha tenido la accesibilidad del transporte público a determinados lugares funcionales, como pueden ser los comercios o las administraciones, condicionado sobre todo por los horarios de apertura de éstos y su compatibilidad con los horarios del transporte público en los espacios rurales o de escasa población (Nutley, 1983; Rodríguez, 1989; Nutley, 1998; Nutley, 2005); o también en ámbitos regionales independientemente del modo de transporte (López Lara, 1990; Niemeier, 1997; Cross \& Nutley, 1999; Yamaguchi, 2007; Neutens, 2012). Así pues, en esta última línea, se profundiza en este trabajo, donde especialmente hemos tomado la accesibilidad de los espacios insulares de menor peso socioeconómico, como son las denominadas islas periféricas de Canarias (Lanzarote, Fuerteventura, La Gomera, El Hierro y La Palma), pues en ellas se localiza la mayor necesidad de viajar a las islas centrales de Tenerife y Gran Canaria ante la ausencia de servicios comerciales, sanitarios o administrativos especializados, siendo muy importante aquí los viajes de retorno en la misma jornada (UTE Eco-CoRe, 2019).

\section{Metodología}

La accesibilidad es difícil de interpretar y explicar en todas sus dimensiones, pues intervienen parámetros físicos, temporales, económicos, etc. (Niemeier, 1997; Farrington, 2007; Bocarejo \& Oviedo, 2012). Conscientes de ello, y a pesar de que en este trabajo nos centramos principalmente en la accesibilidad temporal, no dejamos de lado la conectividad física y económica, variables estas últimas que sobre todo analizamos desde el punto de vista de la complementariedad entre los modos aéreos y marítimos en Canarias y al que también le vamos a proporcionar un peso importante dentro del trabajo, ya que explican, a grandes rasgos, el comportamiento de la demanda condicionada por la accesibilidad de cada modo de transporte. 
Pero como decimos, el análisis de la conectividad, los tiempos de viaje, los costes globales de desplazamiento, etc., recopilando información de los operadores interinsulares, nos van a acercar a los hinterland de cada modo de transporte y, a su vez, a la importancia que tiene para una mejora de la accesibilidad, los horarios de los operadores. Es más, creemos que el análisis de estos últimos es la herramienta básica para justificar la integración territorial del archipiélago, incluso más que las tarifas aéreas como expondremos. Sin embargo, a la accesibilidad temporal no se le ha prestado tanta atención como la conectividad física o la económica, aunque como destacaremos, extremadamente importante para las islas, pues por ejemplo el tiempo extra conlleva un sobrecoste para los viajes de negocios de las empresas, como lo demuestra el hecho de que el 94\% de la economía canaria realiza unos consumos intermedios en agencias de viajes y hostelería más elevados que los correspondientes a los del conjunto nacional (UTE Eco-CoRe, 2019).

Pues bien, para el análisis de la red interinsular aérea de Canarias, vamos a considerar los primeros 21 años correspondientes al Siglo XXI (2000-2020), y siempre tomando las temporadas de programación de invierno, no afectadas por el incremento de la oferta y la demanda del estío. Aun disponiendo de los datos de la temporada invernal 2020-2021, de manera intencionada la hemos obviado, ya que está notoriamente sesgada por la pandemia de la COVID-19. Desde el punto de vista metodológico estudiamos algunos parámetros básicos que nos acercan a las características de la red, como las distancias físicas y las frecuencias marítimas y aéreas, la disponibilidad de asientos, las tarifas, pero en especial en este artículo vamos a centrarnos en los horarios de los operadores, en tanto que los consideramos muy importantes como decíamos.

En efecto, la accesibilidad horaria o "disponibilidad de tiempo" en el destino, condicionada en gran medida por los horarios aéreos, es importante porque va a condicionar la probabilidad de pernoctación en la isla visitada, con los consiguientes costes de alojamiento, dietas alimenticias y la pérdida de horas laborales, por lo que en última instancia el tiempo se traduce en un valor económico perfectamente integrado en la tarifa global del desplazamiento. ${ }^{1}$

1 En la Resolución de 30 de julio de 1998, por el que se aprobaban las obligaciones de servicio público interinsulares de Canarias, se recogió en el Anexo, apartado III, que en las rutas interinsulares "los horarios deberán permitir a los usuarios efectuar una ida y vuelta en el mismo día". En 2006 y 2011, en este último caso para las tres rutas de más débil tráfico, se renuevan dichas obligaciones de servicio público, reflejándose aquí también las alusiones a la planificación horaria. 
Es fundamental también que las frecuencias tengan un cierto grado de dispersión a lo largo de la jornada, para así facilitar los movimientos en que no es necesario permanecer en el destino todo el día. Pero en general, se constata que en la red interinsular canaria los desplazamientos de ida y vuelta en la misma jornada son muy altos, ya que rondan el 45 \% entre las dos islas centrales y llegan a un 23 en los movimientos realizados desde las islas periféricas (Murillo Fort, 1992), mientras que para otras fuentes más recientes estos porcentajes son del 48 y del $27 \%$ respectivamente (Gobierno de Canarias, 2011), con lo que se denota la importancia de la planificación horaria. Si a ello le añadimos que, en gran medida, la red interinsular aérea es utilizada en un porcentaje muy alto por los residentes, pues el $86 \%$ de los viajeros interinsulares en 2018 se desplazó en calidad de residente en las islas (Ramos Pérez, 2020) -y además en ascenso, pues un año más tarde ya era del 87,5 \% (AIREF, 2020)-, deducimos la relevancia de este sistema de transporte para los canarios. A su vez, esta importancia es aún mayor entre los residentes en las islas periféricas, carentes de muchos servicios, pues teóricamente los habitantes de El Hierro o de La Palma, viajaron cada uno de ellos en 2011, más de seis y 4,5 veces respectivamente, frente a los ciudadanos de Gran Canaria que solo alcanzaron 0,90 viajes por residente. $^{2}$

Pues bien, como decíamos, para el cálculo de la accesibilidad horaria en el modo aéreo, hemos tomado como referencia las programaciones invernales desde 2000 a 2020 (21 años) y, más en concreto, los cinco días laborales de la última semana del mes de enero. Se ha considerado este periodo como el más representativo, ya que no está afectado por los incrementos de frecuencias -y consiguiente modificación de horarios-, por motivos de ocio, ni tampoco por eventos culturales, festivos, etc. que, puntualmente, pudieran tergiversar los datos finales de unas rutas frente a otras. Tomamos los días laborales como ejemplo de caso, pues fundamentalmente consideramos los desplazamientos con un fin administrativo o comercial, insertándose dentro de las horas más frecuentes del día en que estos establecimientos permanecen abiertos al público. Además es en estas jornadas, donde se concentra la mayor preocupación de los pasajeros por la disponibilidad de tiempo en los nodos de destino, frente a los fines de semana donde los viajes motivados por el ocio son mayores. Esto se demuestra por ejemplo en la escasa demanda de las frecuencias aéreas de las primeras horas de la mañana, en especial en la jornada dominical, frente a todo lo contrario en los cinco días laborales de la semana (Hernández Luis, 2003).

2 Datos deducidos sobre residentes con bonificación según isla de empadronamiento (Ramos Pérez, 2020). 
En determinadas ocasiones, las primeras y/o últimas frecuencias de un día laboral y dentro de una determinada ruta, no se efectuaban exactamente a la misma hora, con lo que se optó por tomar los horarios más frecuentes (tres días frente a dos por ejemplo).

La accesibilidad horaria propuesta se ha plasmado en el concepto de "disponibilidad de tiempo" en un sistema puerta-puerta entre capitales insulares, contabilizando siempre el tiempo disponible en las islas de destino que es el que realmente nos interesa.

El resultado de este estudio presenta pues un modelo teórico, en el que los viajeros se desplazan en las diferentes rutas -en uno y otro sentido-, tomando siempre la primera frecuencia disponible del día y regresando al nodo, o isla de origen, en la última. Todo ello independientemente de que pueda existir asiento disponible o no, pues como decimos, se trata de un modelo teórico, donde solo se consideran, dentro de una misma ruta, los horarios primero y último en cada sentido, buscando así el mayor tiempo disponible en los destinos.

La "disponibilidad de tiempo" se traduce al final en porcentajes para así mejorar la calidad expositiva, resultado de deducir los bloques de tiempo empleados en el transporte (aéreo y terrestre en destino) más el de la facturación y transporte terrestre a la vuelta. Todo ello en función del horario administrativo (08.00-15.00 horas, es decir, 420 minutos) y del comercial (09.00-13.00 y 16.00-20.00 horas, esto es, 480 minutos). Por tanto, un 100 por cien de "disponibilidad de tiempo" en horario administrativo de un residente de una isla en otra, significa que éste puede llegar a la capital insular de la isla visitada antes de las 08.00 horas y la puede abandonar después de las 15.00. El cálculo de ambas disponibilidades de tiempo durante los 21 años considerados, ha supuesto la realización de dos matrices (una para la disponibilidad de horario administrativo y otra para la comercial), cada una con 462 resultados, totalizando unos 924. Igualmente, habría que destacar una intensa labor personal de seguimiento anual de los horarios de las seis compañías aéreas que han operado regularmente entre las Islas Canarias durante estos años (Air Europa, Atlantic Airways, Binter Canarias, Canaryfly, Islas Airways y NAYSA), por lo que los resultados aquí expuestos son la consecuencia del seguimiento anual de dos décadas para su completa recopilación.

Los bloques de tiempo de un viajero sin equipaje, como situación más habitual de un pasajero commuter interinsular, donde además se intenta maximizar la disponibilidad de tiempo en destino, razón por la cual se excluye el transporte en autobús en favor del taxi o coche de alquiler, se han considerado como sigue: 
Tabla 1. Bloques de tiempo teóricos en "destino" generados en los desplazamientos aéreos hasta las capitales insulares de las Islas Canarias (en minutos)

\begin{tabular}{|c|c|c|c|}
\hline Líneas & $\begin{array}{c}\text { Acceso terrestre } \\
\text { hasta la capital } \\
\text { insular (ida)* }\end{array}$ & $\begin{array}{c}\text { Acceso terrestre } \\
\text { hasta el aeropuerto } \\
\text { (vuelta)* }\end{array}$ & $\begin{array}{l}\text { Facturación, } \\
\text { seguridad y } \\
\text { embarque }\end{array}$ \\
\hline Tenerife-El Hierro & 15 & 15 & 40 \\
\hline El Hierro-Tenerife & 30 & 30 & 40 \\
\hline Tenerife-La Palma & 15 & 15 & 40 \\
\hline La Palma-Tenerife & 30 & 30 & 40 \\
\hline Tenerife-La Gomera & 45 & 45 & 40 \\
\hline La Gomera-Tenerife & 30 & 30 & 40 \\
\hline Tenerife-Gran Canaria & 30 & 30 & 40 \\
\hline Gran Canaria-Tenerife & 30 & 30 & 40 \\
\hline Tenerife-Fuerteventura & 15 & 15 & 40 \\
\hline Fuerteventura-Tenerife & 30 & 30 & 40 \\
\hline$\overline{\text { Tenerife-Lanzarote }}$ & 15 & 15 & 40 \\
\hline Lanzarote-Tenerife & 30 & 30 & 40 \\
\hline Gran Canaria-La Palma & 15 & 15 & 40 \\
\hline La Palma-Gran Canaria & 30 & 30 & 40 \\
\hline Gran Canaria-Fuerteventura & 15 & 15 & 40 \\
\hline Fuerteventura-Gran Canaria & 30 & 30 & 40 \\
\hline Gran Canaria-Lanzarote & 15 & 15 & 40 \\
\hline Lanzarote-Gran Canaria & 30 & 30 & 40 \\
\hline
\end{tabular}

Nota: * En taxi o vehículo de alquiler.

Fuente: elaboración propia

Figura 1. Ejemplo del tiempo disponible en Santa Cruz de Tenerife de un residente en La Gomera que se desplazaba en avión (enero de 2020)

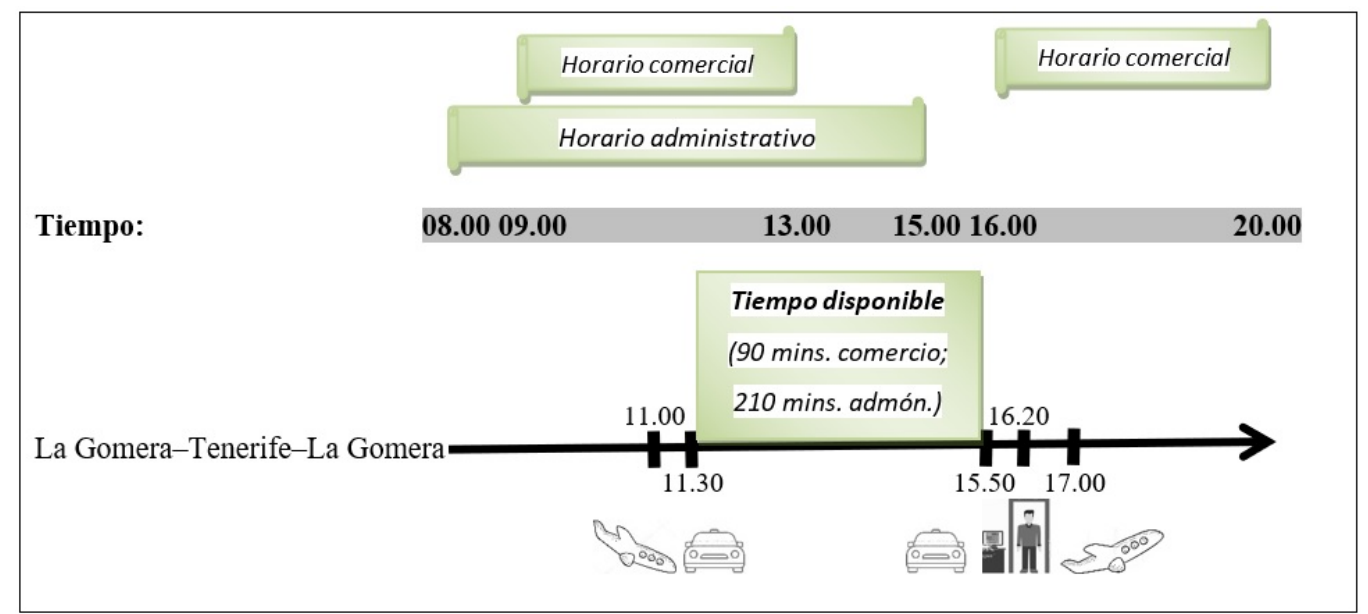

Fuente: elaboración propia a partir de Horarios de Binter Canarias 
Por último, solo se han considerado aquellas líneas en las que existen dos o más frecuencias diarias en cada sentido, pues éste es el requisito indispensable para poder efectuar la ida y vuelta en la misma jornada. Por tanto, se excluyen en ciertos años las rutas Gran Canaria-El Hierro; Gran Canaria-Tenerife Sur, así como Gran Canaria-La Gomera, línea inoperativa desde 2010 inclusive.

Con estas acotaciones al problema, eliminamos prácticamente cualquier atisbo de sesgo, que unido a la alta regularidad de las conexiones interinsulares (Ministerio de Fomento, 2013), descartan en más de un $94 \%$ las situaciones excepcionales en que no se cumplen los horarios por temas de averías técnicas, inclemencias meteorológicas, retrasos en general, etc. En cualquier caso, las excepcionalidades estarían más sujetas a las congestiones en tierra, sobre todo en las carreteras que conectan los aeropuertos de Gran Canaria y Tenerife con sus capitales insulares respectivas y que entran dentro de la imprevisibilidad, debido a que estas están vinculadas sobre todo a incidentes en las vías.

\section{Breve marco territorial de Canarias}

Canarias ha aumentado su población un 120 \% en los últimos 50 años, hasta sobrepasar los 2.1 millones de habitantes en 2019 (ISTAC, 2020). Ello, unido al incremento de la renta y el trasvase de población activa desde el sector primario al terciario, ha favorecido un aumento espectacular de la movilidad interinsular - tanto marítima como aérea-, solo ensombrecida por determinadas crisis profundas como la de 2008 y la de la COVID-19 en 2020.

No obstante, la distribución de la población y de la riqueza dentro del archipiélago varía considerablemente. Esta desigual distribución tiene consecuencias notables para el transporte exterior, pues en gran medida, el hinterland de cada isla condiciona la demanda de transporte. Pero también es verdad que algunas islas, como La Gomera o El Hierro, necesitan de unos niveles mínimos de servicio y que, en principio, por su escaso volumen demográfico y económico, no justificarían una mejora de la oferta aérea con estas islas, si bien es muy importante la citada mejora en aras de la integración territorial del archipiélago. 
Tabla 2. Principales macromagnitudes de las Islas Canarias en 2019

\begin{tabular}{l|c|c|c}
\hline Islas & Población & Turistas* & Producto Interior Bruto (\%) \\
\hline Tenerife & 917841 & 5889454 & 41 \\
\hline Gran Canaria & 851231 & 4272615 & 41 \\
\hline Lanzarote & 152289 & 3065575 & 7 \\
\hline Fuerteventura & 116886 & 2023196 & 6 \\
\hline La Palma & 82671 & 343680 & 4 \\
\hline La Gomera & 21503 & 114150 & 0.5 \\
\hline El Hierro & 10968 & s.d. & 0.5 \\
\hline Total & 2153389 & 15708670 & 100 \\
\hline
\end{tabular}

Nota: * A ello habría que añadirle los cruceristas que, en 2019, alcanzaron los 2.553.397. No obstante, la estancia de estos suele ser de una jornada diurna, por lo que se les califica de excursionistas.

Fuente: elaboración propia a partir de Instituto Canario de Estadística (2020)

Así pues, Tenerife y Gran Canaria concentraban en 2019 el 82 \% de la población, al igual que del Producto Interior Bruto (PIB). El restante $18 \%$ también se distribuía desigualmente, pues si algo más del $16 \%$ de la población se localizaba en las islas de Lanzarote, La Palma y Fuerteventura, el restante porcentaje, ni siquiera el $2 \%$, se repartía entre La Gomera y El Hierro. El PIB difería muy poco de estos valores.

En síntesis, estos parámetros condicionan la disponibilidad de servicios públicos y privados en las diferentes islas, de tal modo que las que presentan menor volumen demográfico y económico se ven supeditadas a disfrutar de esos servicios en las islas centrales, utilizando como soporte la red marítima y aérea, de ahí la alta recurrencia de los viajes en el sentido isla periférica - central con todas sus consecuencias.

\section{El papel del transporte aéreo en Canarias}

Como ya se argumentó más arriba, el transporte aéreo es fundamental para el desarrollo económico mundial (Button \& Taylor, 2000), pero más lo es para los territorios insulares, debido sobre todo a la fragmentación territorial y también la lejanía (Deidda, 2016). Estas debilidades solo pueden ser solventadas con un medio de transporte rápido, como es el avión, para el transporte de pasajeros y mercancías urgentes.

Multitud de estudios han subrayado el protagonismo que adquiere el transporte en las Islas (Murillo Fort, 1992; Eurisles, 1996; Gobierno de Canarias, 1998; Fundación Tomillo, 2001; Ramos Pérez, 2001; Hazledine \& Collins, 2011; Ramos Pérez, 2020). No obstante, en una sociedad donde la información, mercancías y viajeros fluyen cada vez con mayor rapidez, el 
transporte aéreo es una pieza fundamental de este engranaje. Además, gran cantidad de archipiélagos dependen casi por completo de la actividad turística (Hawái, Seychelles, Islas Vírgenes, Baleares, etc.) y, debido a su lejanía de los principales centros emisores del turismo, el transporte aéreo se constituye en un baluarte esencial para su desarrollo.

En este contexto, el transporte aéreo interinsular en Canarias aumentó considerablemente en los últimos años, hasta duplicarse entre 1998 y 2007. Solo retrocedió ostensiblemente entre este último año y 2013 debido a la fuerte crisis acaecida en 2008 (Ramos Pérez, 2015) y, muy recientemente, con la COVID-19. No obstante, el incremento más fuerte de todo el período se registró en tan solo tres años (2017-2019), ya que aumentó el 50 \% con respecto a 2016. Gran parte de este crecimiento se debió al cambio en la bonificación al residente, al pasar del 50 al $75 \%$ en 2017, sin obviar una mejora de la economía en general y, a pesar de la fuerte competitividad marítima.

\section{Figura 2. Transporte regular de pasajeros aéreos interinsulares en Canarias (1975-2019)}

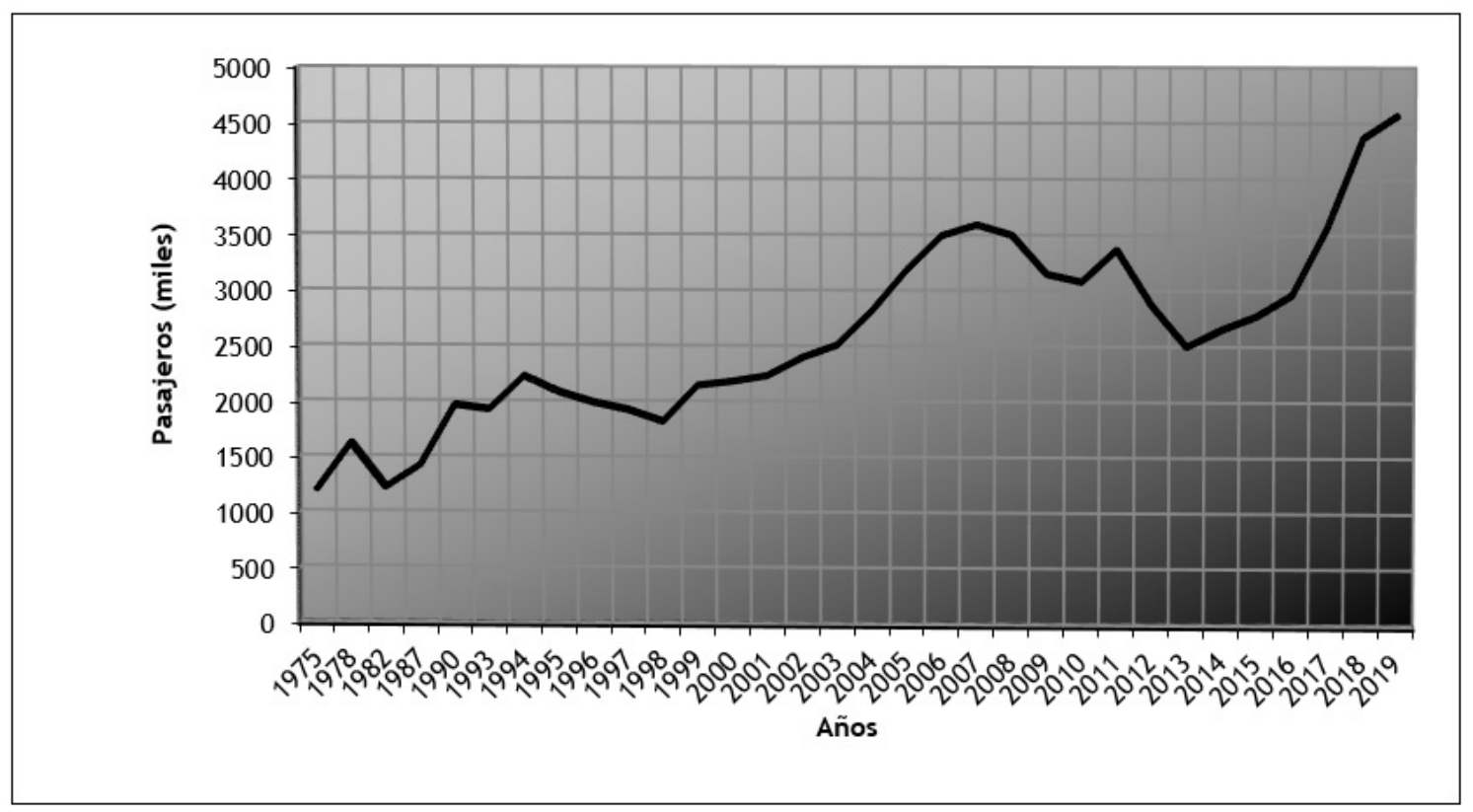

Fuente: elaboración propia a partir de Aeropuertos Españoles y Navegación Aérea (2020)

Como decimos, la competencia marítima también ha jugado su papel, hasta desplazar incluso más viajeros en comparación con el aéreo. Así, en 2019, la red marítima transportó más de 5,8 millones de pasajeros, alcanzando el $56 \%$ del total, aunque bien es verdad que un segmento significativo de la demanda marítima la podríamos calificar como "cautiva" de portar consigo el 
vehículo privado. ${ }^{3}$ Entre ambos modos de transporte, el movimiento de pasajeros superó los 10 millones, dando lugar a una de las redes de transporte insulares más importantes del Mundo.

Sin embargo, creemos que más que una competencia entre ambos modos de transporte, debemos hablar de una complementariedad, pues el hecho de transportar consigo el vehículo, ya excluye la utilización del avión (García Gordillo, 1983). Pero no es el único factor, pues en algunas rutas no existe conexión directa de un modo de transporte u otro. Así por ejemplo, en las rutas cuya distancia física y en tiempo de navegación es muy corta, no existe conexión aérea directa (caso de Lanzarote - Fuerteventura), mientras que en una situación inversa, son las navieras las que no ofrecen este servicio (ejemplo de Tenerife - Fuerteventura). También hay que incluir animadversiones a un medio de transporte u otro, los horarios, el complejo entramado de seguridad en los embarques de los aeropuertos, incluso el sistema tarifario, aunque sin grandes diferencias entre ambos modos de transporte, etc. $Y$, por supuesto, la consideración del origen y destino final (puerta - puerta) dentro de cada isla. Por tanto, confluyen muchos factores en la elección de un modo de transporte u otro y que, por su complejidad y profundidad, trascienden más allá de los objetivos de este trabajo.

Pero por norma general, entre las islas en las que los buques superan las dos horas de navegación, el transporte aéreo supera con creces al marítimo. Así, en el año 2019, el transporte marítimo entre Lanzarote y Gran Canaria solo desplazó unos 196 mil pasajeros, frente a los más de 800 mil aéreos. Por el contrario, entre las dos islas centrales, con buques rápidos y travesías de poco más de una hora, los viajeros aéreos solo alcanzaron el 42 \% de dicha ruta.

3 Ejemplo de ello es que en 2019 y en la ruta Agaete (Gran Canaria) - Santa Cruz de Tenerife, cada pasajero transportó 0,37 vehículos, mientras que entre Las Palmas de Gran Canaria y Arrecife (Lanzarote), fue de 0,43. 
Tabla 3. Movimiento de pasajeros regulares en rutas directas en Canarias en 2019 (ida y vuelta)

\begin{tabular}{|c|c|c|c|c|c|}
\hline \multirow{2}{*}{ Líneas } & \multicolumn{2}{|c|}{ Aéreos } & \multicolumn{2}{|c|}{ Marítimos } & \multirow{2}{*}{ Total } \\
\hline & Pasajeros & Cuota (\%) & Pasajeros & Cuota (\%) & \\
\hline Gran Canaria - Tenerife & 1147945 & 41,87 & 1593938 & 58,13 & 2741883 \\
\hline Tenerife - La Gomera & 60782 & 4,19 & 1391116 & 95,81 & 1451898 \\
\hline $\begin{array}{l}\text { Gran Canaria - } \\
\text { Fuerteventura }\end{array}$ & 665010 & 49,75 & 671815 & 50,25 & 1336825 \\
\hline Lanzarote - Fuerteventura & $\begin{array}{ll}---- \\
\end{array}$ & 0,00 & 1077382 & 100,00 & 1077382 \\
\hline Tenerife - La Palma & 764879 & 73,97 & 269173 & 26,03 & 1034052 \\
\hline Gran Canaria - Lanzarote & 803358 & 80,36 & 196371 & 19,64 & 999729 \\
\hline Lanzarote - La Graciosa & ---- & 0,00 & 446654 & 100,00 & 446654 \\
\hline Tenerife - Lanzarote & 394082 & 100,00 & --- & 0,00 & 394082 \\
\hline Tenerife - El Hierro & 211285 & 55,94 & 166407 & 44,06 & 377692 \\
\hline Tenerife - Fuerteventura & 298114 & 100,00 & ---- & 0,00 & 298114 \\
\hline Gran Canaria - La Palma & 164241 & 100,00 & ---- & 0,00 & 164241 \\
\hline Gran Canaria - El Hierro & 55876 & 100,00 & ---- & 0,00 & 55876 \\
\hline Gran Canaria - La Gomera & 16521 & 100,00 & ---- & 0,00 & 16521 \\
\hline TOTAL & 4582093 & 44,08 & 5812856 & 55,92 & 10394949 \\
\hline
\end{tabular}

Fuente: elaboración propia a partir de Departamentos de estadística de Binter Canarias, Canaryfly, Naviera Armas, Fred. Olsen, Líneas Marítimas Romero y Biosfera Express

Figura 3. Pasaje aéreo interinsular y en líneas regulares en Canarias en 2019

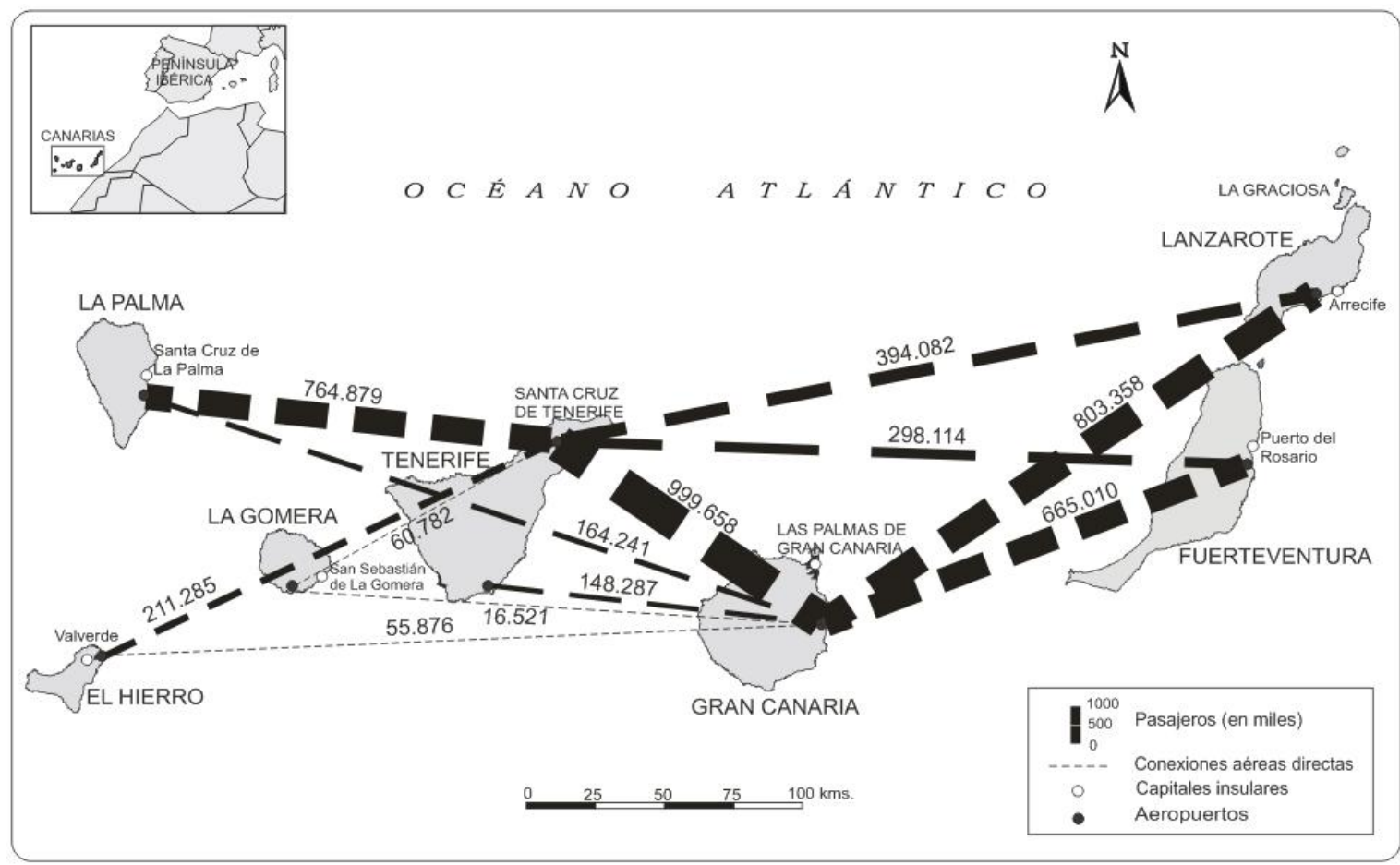

Fuente: elaboración propia a partir de Estadísticas de AENA, Ministerio de Fomento (2020) 
En suma, el transporte aéreo interinsular en Canarias es el modo más accesible para las rutas más distantes y también porque la cuota de frecuencias representa casi dos tercios de todas las conexiones diarias, suponiendo además una variedad horaria más flexible y, por ende, mejor adaptada a la demanda final

\section{Análisis de la accesibilidad aérea interinsular}

La mejora de la accesibilidad en una red de transporte interinsular se ha considerado un factor clave de desarrollo de estos territorios, incluyendo en ello un incremento de las frecuencias, asientos, mejora del sistema tarifario, etc. (Hoyle, 1999; Ranély Vergé-Dépré, 2005, Hernández Luis, 2018). Si bien es cierto que, sobre todo con la pandemia de 2020, se ha puesto de manifiesto el carácter prescindible de algunos desplazamientos (reuniones de negocios y de la administración, docencia, etc.), determinados motivos como son los sanitarios, reparaciones de maquinarias, sector de ventas, etc., exigen una óptima accesibilidad, sobre todo en distancias cortas que pueden permitir el round trip en la misma jornada, como es el caso de gran parte de las redes de transporte interinsulares. Es aquí cuando el mantenimiento y mejora de la accesibilidad aérea debe ser un tema prioritario, pues por ejemplo, la atención sanitaria especializada en Canarias se localiza en los hospitales de referencia de las islas centrales, constituyendo de facto un problema de equidad en el sistema sanitario, sobre todo para la envejecida población de las islas periféricas occidentales (Abásolo Alessón, 2008). Igualmente, el mayor coste en el que incurren las empresas canarias respecto a las peninsulares como consecuencia de los déficits de accesibilidad interinsular y con la Península, está relacionado en un alto porcentaje con la pérdida de jornadas debido a la mayor duración de los viajes entre islas, muchas veces por problemas con los horarios, además de los gastos de estancia y manutención y facturación por persona y día (UTE Eco-CoRe, 2019). En este sentido, tomamos algunos datos relevantes de este estudio, referido a 2016 y a la demanda de viajes interinsulares, que demuestra la necesidad de actuar en la mejora de la accesibilidad entre islas:

1. El $25 \%$ de las empresas canarias (es decir, 15873), precisaron que sus trabajadores realizaran viajes interinsulares por motivo de negocios.

2. Los viajes interinsulares por este motivo se elevaron a 468569.

3. El $63 \%$ de los viajes interinsulares por negocios lo fue en avión.

4. El $30 \%$ de las empresas encuestadas consideró que sin fragmentación territorial, la duración de los viajes interinsulares se reduciría un día. 
5. Las jornadas perdidas por los trabajadores de las empresas canarias debido al incremento de la duración de los viajes fue de 441133 (el $60 \%$ de ellos imputable a los viajes interinsulares).

6. El sobrecoste por incremento de la duración de los viajes fue de 243592210 euros (82 \% imputable a la pérdida de tiempo; 10 \% por la manutención; y 8 \% al alojamiento).

Bien es cierto que el Estado ha arbitrado medidas para paliar esta vulnerabilidad de las islas, como por ejemplo la sustitución del IVA (Impuesto sobre el Valor Añadido) por el IGIC (Impuesto General Indirecto Canario), con tipos generales del 21 y 7 \% respectivamente. Aun así y a partir de estos datos tan reveladores del coste de la insularidad en Canarias, es necesario abordar la mejora de la accesibilidad aérea. Vamos a analizar esta desde varios puntos de vista: conectividad, frecuencias, tarifas globales puerta - puerta y, en especial, los horarios.

\section{Tabla 4. Distancias físicas de las líneas interinsulares canarias actualmente operativas entre "capitales insulares" (en kilómetros)}

\begin{tabular}{|c|c|c|c|}
\hline Líneas & Avión & Barco & $\begin{array}{c}\text { Diferencia \% avión - } \\
\text { barco }\end{array}$ \\
\hline Gran Canaria - Tenerife & $148 / 202^{*}$ & $101 / 107^{\star}$ & $47 / 38$ \\
\hline Tenerife - La Gomera & 147 & 115 & 28 \\
\hline Gran Canaria - Fuerteventura & 192 & $192 / 187^{\star}$ & $0 / 3$ \\
\hline Lanzarote - Fuerteventura & ---- & 82 & ---- \\
\hline Tenerife - La Palma & 162 & 202 & -20 \\
\hline Gran Canaria - Lanzarote & 243 & 211 & 15 \\
\hline Lanzarote - La Graciosa & ---- & 42 & ---- \\
\hline Tenerife - Lanzarote & 295 & 315 & -6 \\
\hline Tenerife - El Hierro & 191 & 197 & -3 \\
\hline Tenerife - Fuerteventura & 275 & $293 / 288^{*}$ & $-6 /-5$ \\
\hline Gran Canaria - La Palma & 277 & 303 & -9 \\
\hline Gran Canaria - El Hierro & 281 & 298 & -6 \\
\hline Gran Canaria - La Gomera & 238 & 217 & 10 \\
\hline
\end{tabular}

Nota: * Estas distancias se corresponden con dos rutas diferentes dentro de una misma línea y modo de transporte. No obstante, el primer dato se corresponde con la ruta de mayor demanda.

Fuente: elaboración propia

Por un lado, las características de los trayectos, sobre todo las distancias, contribuyen a explicar por qué en unas rutas predomina un medio de transporte u otro. La siguiente es una tabla donde se exponen las distancias físicas entre capitales insulares como punto de la matriz de salida o llegada (puerta - puerta). Es en estos enclaves de todas las islas, exceptuando en La Palma 
parcialmente, donde se concentra la mayor población y en gran medida también la actividad económica y administrativa y, por ende, mayor posibilidad de que se convierta en nodo de origen y de destino. Por tanto, en un trayecto puerta-puerta, también se toman las distancias terrestres, resultando la conclusión de que toda distancia que presente más de 120 kilómetros de ida, ya empieza a favorecer al modo aéreo.

Tabla 5. Frecuencias directas marítimas y aéreas medias y por día de pasajeros con carácter regular entre las Islas Canarias en enero de 2020 (ida y vuelta)

\begin{tabular}{|c|c|c|c|c|c|}
\hline \multirow{2}{*}{ Líneas } & \multicolumn{2}{|c|}{ Aéreas } & \multicolumn{2}{|c|}{ Marítimas } & \multirow{2}{*}{ Total } \\
\hline & Frecuencias & Cuota \% & Frecuencias & Cuota \% & \\
\hline $\begin{array}{l}\text { Gran Canaria - Tenerife - Gran } \\
\text { Canaria }\end{array}$ & 52,86 & 65,14 & 28,29 & 34,86 & 81,15 \\
\hline Tenerife - La Palma - Tenerife & 40,29 & 90,68 & 4,14 & 9,32 & 44,43 \\
\hline $\begin{array}{l}\text { Gran Canaria - Lanzarote - } \\
\text { Gran Canaria }\end{array}$ & 35,14 & 86,02 & 5,71 & 13,98 & 40,85 \\
\hline $\begin{array}{l}\text { Gran Canaria - Fuerteventura - } \\
\text { Gran Canaria }\end{array}$ & 32,28 & 81,00 & 7,57 & 19,00 & 39,85 \\
\hline $\begin{array}{l}\text { Lanzarote - Fuerteventura - } \\
\text { Lanzarote }\end{array}$ & 0,00 & 0,00 & 33,28 & 100,00 & 33,28 \\
\hline $\begin{array}{l}\text { Lanzarote - La Graciosa - } \\
\text { Lanzarote }\end{array}$ & 0,00 & 0,00 & 32,00 & 100,00 & 32,00 \\
\hline Tenerife - Lanzarote - Tenerife & 19,14 & 100,00 & 0,00 & 0,00 & 19,14 \\
\hline $\begin{array}{l}\text { Tenerife - La Gomera - } \\
\text { Tenerife }\end{array}$ & 4,00 & 34,13 & 11,72 & 65,87 & 15,72 \\
\hline Tenerife - El Hierro - Tenerife & 12,57 & 84,59 & 2,29 & 15,41 & 14,86 \\
\hline $\begin{array}{l}\text { Tenerife - Fuerteventura - } \\
\text { Tenerife }\end{array}$ & 14,00 & 100,00 & 0,00 & 0,00 & 14,00 \\
\hline $\begin{array}{l}\text { Gran Canaria - La Palma - } \\
\text { Gran Canaria }\end{array}$ & 8,86 & 100,00 & 0,00 & 0,00 & 8,86 \\
\hline $\begin{array}{l}\text { Gran Canaria - El Hierro - } \\
\text { Gran Canaria }\end{array}$ & 3,71 & 100,00 & 0,00 & 0,00 & 3,71 \\
\hline $\begin{array}{l}\text { Gran Canaria - La Gomera - } \\
\text { Gran Canaria }\end{array}$ & 0,57 & 100,00 & 0,00 & 0,00 & 0,57 \\
\hline TOTAL & 223,42 & 64,13 & 125,00 & 35,87 & 348,42 \\
\hline
\end{tabular}

Fuente: elaboración propia a partir de Binter Canarias, Canaryfly, Fred. Olsen,

Naviera Armas, Líneas Marítimas Romero y Biosfera Express

En casi todas estas rutas directas opera tanto el barco como el avión, pero es este último el que más frecuencias diarias ofrece, si exceptuamos la línea entre Tenerife y La Gomera. De la importante cifra de 350 frecuencias diarias entre islas de ambos modos de transporte en enero de 2020, el $65 \%$ eran aéreas, elevándose a más del 80 \% para las rutas de mayor distancia. 
Todo ello corrobora la idea antes expuesta de que a mayor distancia -incluso con buques rápidos-, el modo aéreo consigue mejores cuotas.

Si como decimos, las distancias físicas ya nos orientan bastante sobre el hinterland por parte de uno y otro medio de transporte, los tiempos de viaje y las franjas horarias de llegada a las capitales insulares, según la primera frecuencia de los operadores marítimos y aéreos, terminan por definir la demanda. De este modo, el límite del transporte marítimo puerta - puerta parece estar en el entorno de los 160-180 minutos de ida, favorecido además porque las llegadas a las capitales insulares se producen en una franja horaria aceptable, incluso antes de las 08.00 horas. Es el caso del corredor Gran Canaria - Tenerife, donde la llegada de los viajeros a la capital es ostensiblemente anterior a la de los pasajeros aéreos (véase Tabla 6). Bien es verdad que en otras rutas como el sentido Gran Canaria - Fuerteventura o Gran Canaria - Lanzarote, esas llegadas se producen notoriamente antes, ya que los buques parten a medianoche, pero aquí el elevado tiempo de transporte, más de un 300 \% con respecto al avión, decanta claramente la balanza a favor del modo aéreo.

Como argumentábamos más arriba, también el coste global del desplazamiento entre las capitales insulares puede ser un condicionante de la elección de un modo de transporte u otro (Hernández Luis, 2000). Si bien es verdad que este no es excesivamente dispar entre ambos modos y, por tanto, a falta de un análisis más profundo, no determina claramente la elección, lo cierto es que para un viaje aéreo de un residente, el monto imputable a los desplazamientos terrestres puede suponer una tarifa mayor que la impuesta por la compañía aérea, en el caso de que se opte por el taxi o un vehículo de alquiler. La gran ventaja del transporte marítimo, aunque no ambiental, es la posibilidad de portar consigo el vehículo privado, disminuyendo considerablemente los costes del transporte en el destino, tanto a la ida como a la vuelta. Por ello tenemos que en varias rutas, como por ejemplo entre Gran Canaria y Tenerife; Tenerife La Gomera; o Gran Canaria - Fuerteventura, el coste del avión más el autobús, frente al del transporte marítimo con vehículo, es prácticamente igual y si a ello le unimos unas distancias físicas y en tiempo de las más aceptables para ambos medios de transporte, se deduce que aquí la aceptación del transporte marítimo es elevada, incluso con mejor cuota frente al avión. Sin embargo, son las rutas de mayor distancia, sobre todo entre isla central y periférica de la otra provincia, siendo necesario además hacer transbordo, donde se detecta una mayor disparidad tarifaria en favor del avión, por lo que en estas, unido al factor mencionado de distancia y tiempo, la cuota aérea es muy alta y la marítima queda relegada a la cautividad de transportar el vehículo privado (véase Tabla 7). 
Tabla 6. Tiempos de viaje reales y primera franja horaria de llegada a destino en días laborales en enero de 2020*

\begin{tabular}{|c|c|c|c|c|}
\hline \multirow[t]{2}{*}{ Líneas } & \multicolumn{2}{|c|}{$\begin{array}{c}\text { Tiempos de viaje puerta - } \\
\text { puerta (en mins.) }\end{array}$} & \multicolumn{2}{|c|}{$\begin{array}{c}\text { Primera hora de llegada a la } \\
\text { "capital insular" de } \\
\text { destino** }\end{array}$} \\
\hline & Avión & Barco & Avión & Barco \\
\hline Gran Canaria - Tenerife & $120 / 160$ & $160 / 160$ & 08.00 & 07.30 \\
\hline Tenerife - Gran Canaria & 120 & $160 / 160$ & 08.00 & 07.55 \\
\hline Tenerife - La Gomera & 135 & 160 & 10.45 & 09.55 \\
\hline La Gomera - Tenerife & 135 & 160 & 11.30 & 08.50 \\
\hline $\begin{array}{l}\text { Gran Canaria - } \\
\text { Fuerteventura }\end{array}$ & 110 & $210 / 470$ & 07.55 & $\begin{array}{c}10.50 / \\
06.40 * * *\end{array}$ \\
\hline $\begin{array}{l}\text { Fuerteventura - Gran } \\
\text { Canaria }\end{array}$ & 110 & $210 / 470$ & 08.05 & $\begin{array}{c}08.40 / \\
16.10 * \star \star\end{array}$ \\
\hline Lanzarote - Fuerteventura & ---- & 110 & ---- & 08.05 \\
\hline Fuerteventura - Lanzarote & ---- & 110 & ---- & 08.05 \\
\hline Tenerife - La Palma & 105 & 260 & 08.15 & 11.10 \\
\hline La Palma - Tenerife & 105 & 260 & 09.00 & 09.00 \\
\hline Gran Canaria - Lanzarote & 115 & 410 & 08.10 & 06.40 \\
\hline Lanzarote - Gran Canaria & 115 & 410 & 08.00 & 16.55 \\
\hline Lanzarote - La Graciosa & ---- & 85 & ---- & 08.35 \\
\hline La Graciosa - Lanzarote & ---- & 85 & ---- & 08.00 \\
\hline Tenerife - Lanzarote & 120 & 680 & 08.10 & ---- \\
\hline Lanzarote - Tenerife & 120 & 700 & 09.45 & 22.00 \\
\hline Tenerife - El Hierro & 110 & 250 & 08.50 & 20.30 \\
\hline El Hierro - Tenerife & 110 & 250 & 10.00 & 17.45 \\
\hline Tenerife - Fuerteventura & 115 & $440 / 680$ & 08.15 & 12.30 \\
\hline Fuerteventura - Tenerife & 115 & $400 / 700$ & 09.45 & 15.30 \\
\hline Gran Canaria - La Palma & 115 & 410 & 08.15 & 22.10 \\
\hline La Palma - Gran Canaria & 115 & 615 & 10.00 & 16.00 \\
\hline Gran Canaria - El Hierro & 120 & 540 & 16.30 & 20.30 \\
\hline El Hierro - Gran Canaria & 120 & 515 & 17.45 & 21.50 \\
\hline Gran Canaria - La Gomera & 140 & 345 & ---- & 10.25 \\
\hline La Gomera - Gran Canaria & 140 & 550 & ---- & 16.00 \\
\hline
\end{tabular}

Notas: * Tiempos de viaje entre "capitales insulares", incluyendo los tiempos medios de transporte terrestre, facturación, medidas de seguridad y rutas aéreas o marítimas, siempre en la embarcación de mayor velocidad.

** Existe una alta probabilidad, no considerada, de congestión viaria en esta primera franja horaria de la mañana en las islas de Tenerife y Gran Canaria.

$\star \star \star$ El primer horario reseñado se corresponde con el de mayor demanda de tráfico.

Fuente: elaboración propia a partir de Binter Canarias, Canaryfly, Fred. Olsen, Naviera Armas, Líneas Marítimas Romero y Biosfera Express 
Tabla 7. Coste del transporte marítimo y aéreo de pasajeros de ida con carácter regular entre las Islas Canarias en enero de 2020 (en euros)*

\begin{tabular}{l|c|c}
\hline Líneas & Avión** & Barco*** \\
\hline Gran Canaria - Tenerife & $19,68 / 24,63 / 67,68$ & $10,45 / 18,59$ \\
\hline Tenerife - La Gomera & $20,41 / 27,06 / 42,41$ & $9,00 / 26,75$ \\
\hline Gran Canaria - Fuerteventura & $26,18 / 30,28 / 73,18$ & $12,75 / 28,50$ \\
\hline Lanzarote - Fuerteventura & ----- & $7,25 / 18,50$ \\
\hline Tenerife - La Palma & $20,16 / 24,31 / 53,16$ & $12,50 / 29,00$ \\
\hline Gran Canaria - Lanzarote & $23,93 / 27,73 / 65,93$ & $19,51 / 34,81$ \\
\hline Lanzarote - La Graciosa & ----- & $3,50 /-----$ \\
\hline Tenerife - Lanzarote & $29,16 / 33,31 / 59,16$ & $25,78 / 54,22$ \\
\hline Tenerife - El Hierro & $21,41 / 25,24 / 55,41$ & $12,38 / 22,88$ \\
\hline Tenerife - Fuerteventura & $27,91 / 36,11 / 62,91$ & $23,87 / 47,74$ \\
\hline Gran Canaria - La Palma & $29,93 / 33,73 / 74,93$ & $26,59 / 48,67$ \\
\hline Gran Canaria - El Hierro & $30,68 / 34,16 / 76,68$ & $22,83 / 41,47$ \\
\hline Gran Canaria - La Gomera & $22,25 / 28,55 / 56,25$ & $16,57 / 48,38$
\end{tabular}

Notas: * Tarifas aplicando el descuento por residente en Canarias. Siempre se toma la tarifa más demandada según los operadores (tarifas "flexible" y "completa" para Binter Canarias y Canaryfly respectivamente; y "básica" y "butaca" para Fred Olsen y Naviera Armas).

** La primera tarifa se corresponde con la denominada "turista" y la segunda incluye además el gasto de desplazamiento terrestre a y desde los aeropuertos en origen y en destino a las capitales insulares en autobús. En la tercera tarifa se considera el taxi en origen y destino, aunque en La Gomera es muy usual tomar el autobús debido a la mayor distancia y su alta eficiencia vinculando las salidas y llegadas con los horarios aéreos.

*** La primera tarifa se corresponde con la butaca de un adulto. La segunda incluye el pasajero más un vehículo tipo turismo normal y de menos de 1,90 metros de alto.

Fuente: elaboración propia a partir de compañías aéreas y navieras operadoras de las líneas interinsulares canarias; compañías de transporte público terrestre

Sin embargo, el impacto del sistema tarifario global en la demanda es muy variable, depende en gran medida de los ingresos de los viajeros y del tiempo empleado en el transporte, máxime si este último se produce dentro de la jornada laboral, cuestión que suele ser muy habitual en las primeras horas del día. También depende, claro está, del valor que cada persona le otorga al mismo (Durán Heras, 2006). Como fuere, el valor del tiempo es muy recomendable que también se compute dentro de la tarifa global (Calmet, 2011; Parras, 2015), tal y como realizamos a continuación, aunque en este caso en función de las categorías profesionales y su nivel de ingresos medios, sobre todo partiendo de la base de los principales usuarios del transporte interinsular, en línea con las categorías descritas en la siguiente tabla: 
Tabla 8. Coste del tiempo de viaje de ida entre las capitales insulares de Canarias según ganancia por hora normal de trabajo y en función de los grupos de ocupación con un perfil de mayor movilidad interinsular en enero de 2020 (en euros) ${ }^{1}$

\begin{tabular}{|c|c|c|c|c|c|c|c|c|}
\hline \multirow[t]{2}{*}{ Líneas } & \multicolumn{2}{|c|}{$\begin{array}{c}\text { Directores y } \\
\text { gerentes }\end{array}$} & \multicolumn{2}{|c|}{$\begin{array}{l}\text { Profesionales } \\
\text { científicos e } \\
\text { intelectuales }\end{array}$} & \multicolumn{2}{|c|}{$\begin{array}{c}\text { Técnicos } \\
\text { profesionales } \\
\text { de apoyo }\end{array}$} & \multicolumn{2}{|c|}{$\begin{array}{l}\text { Todas las } \\
\text { categorías }^{3}\end{array}$} \\
\hline & Avión & Barco $^{2}$ & Avión & Barco $^{2}$ & Avión & Barco $^{2}$ & Avión & Barco $^{2}$ \\
\hline Gran Canaria - Tenerife $^{4}$ & 47,78 & 63,71 & 35,42 & 47,23 & 23,54 & 31,39 & 20,46 & 27,28 \\
\hline Tenerife - La Gomera ${ }^{4}$ & 49,77 & 63,71 & 36,90 & 47,23 & 24,52 & 31,39 & 21,31 & 27,28 \\
\hline Gran Canaria - Fuerteventura $^{4}$ & 43,80 & 83,61 & 32,47 & 61,98 & 21,58 & 41,19 & 18,76 & 35,81 \\
\hline Lanzarote - Fuerteventura $^{4}$ & --- & 43,80 & --- & 32,47 & --- & 21,58 & --- & 18,76 \\
\hline Tenerife - La Palma ${ }^{4}$ & 41,81 & 103,52 & 30,99 & 76,74 & 20,60 & 51,00 & 17,90 & 44,33 \\
\hline Gran Canaria - Lanzarote ${ }^{4}$ & 45,79 & 163,25 & 33,94 & 121,02 & 22,56 & 80,43 & 19,61 & 69,91 \\
\hline Lanzarote - La Graciosa $^{4}$ & --- & 33,84 & --- & 25,09 & --- & 16,67 & --- & 14,49 \\
\hline Tenerife - Lanzarote & 47,78 & 270,75 & 35,42 & 200,71 & 23,54 & 133,39 & 20,46 & 115,94 \\
\hline Lanzarote - Tenerife & 47,78 & 278,72 & 35,42 & 206,62 & 23,54 & 137,32 & 20,46 & 119,35 \\
\hline Tenerife - El Hierro & 43,80 & 99,54 & 32,47 & 73,79 & 21,58 & 49,04 & 18,76 & 42,63 \\
\hline El Hierro - Tenerife & 43,80 & 99,54 & 32,47 & 73,79 & 21,58 & 49,04 & 18,76 & 42,63 \\
\hline Tenerife - Fuerteventura & 45,79 & 175,19 & 33,94 & 129,87 & 22,56 & 86,31 & 19,61 & 75,02 \\
\hline Fuerteventura - Tenerife & 45,79 & 159,27 & 33,94 & 118,07 & 22,56 & 78,47 & 19,61 & 75,02 \\
\hline Gran Canaria - La Palma & 45,79 & 163,25 & 33,94 & 121,02 & 22,56 & 80,43 & 19,61 & 69,91 \\
\hline La Palma - Gran Canaria & 45,79 & 244,87 & 33,94 & 181,53 & 22,56 & 120,64 & 19,61 & 104,86 \\
\hline Gran Canaria - El Hierro & 47,78 & 215,01 & 35,42 & 159,39 & 23,54 & 105,93 & 20,46 & 92,07 \\
\hline El Hierro - Gran Canaria & 47,78 & 205,06 & 35,42 & 152,01 & 23,54 & 101,03 & 20,46 & 87,81 \\
\hline Gran Canaria - La Gomera & 55,74 & 137,37 & 41,32 & 101,83 & 27,46 & 67,68 & 23,87 & 58,82 \\
\hline La Gomera - Gran Canaria & 55,74 & 218,99 & 41,32 & 162,34 & 27,46 & 107,89 & 23,87 & 93,78 \\
\hline
\end{tabular}

1 Los datos para Canarias de ganancia por hora normal de trabajo se refieren a 2018.

2 En transporte marítimo siempre se toma la ruta, dentro de una conexión interinsular entre dos o más islas, con mayor demanda, coincidente con la que emplea menos tiempo de travesía.

3 Se incluyen las restantes siete categorías laborales, totalizando diez. La ganancia media por hora normal de trabajo en Canarias fue de 10,23 euros en 2018, siendo de 23,89; 17,71; y 11,77 euros en el primero, segundo y tercer grupo de ocupación reseñados en esta tabla.

4 En estas rutas solo se indica un sentido porque los datos del sentido inverso son exactamente iguales.

Fuente: elaboración propia a partir de la Encuesta Anual de Estructura Salarial (2020), el INE y operadores aéreos y marítimos en las rutas interinsulares de Canarias

Así pues, del análisis del coste del tiempo de viaje entre capitales insulares, se obtiene que siempre es mayor en el modo marítimo, porque evidentemente los tiempos de desplazamiento son mayores, aunque depende del origen y destino final. De este modo tenemos que a mayor salario de los viajeros, el impacto económico del tiempo de transporte es evidentemente mayor, por lo que entre los directivos y en el puerta - puerta del corredor entre capitales de Gran Canaria y Tenerife, la diferencia del coste del tiempo de ida es del 33 \% y casi 16 euros en favor 
del avión, mientras que este valor se reduce a la mitad en la categoría profesional de los técnicos de apoyo, con más probabilidades que estos últimos se decanten, a priori, por el transporte marítimo.

Por lo que respecta a la disponibilidad de asientos en las horas de mayor demanda y también con mayor separación horaria, permitiendo el máximo tiempo disponible en el destino dentro de una jornada, hemos procedido a realizar la siguiente tabla donde solo se muestran las rutas directas que ofrecen algún asiento en esas horas, además de las que presentan dos o más frecuencias diarias de ida, esto último acorde con los requisitos mínimos de calidad que ya reconocían las Obligaciones de Servicio Público aéreas de Canarias de 30 de julio de 1988 y su revisión de 21 de julio de 2006 :

Tabla 9. Disponibilidad de asientos aéreos interinsulares en Canarias por día laboral en las horas de mayor demanda (programación invernal 2019-2020)*

\begin{tabular}{|c|c|c|}
\hline Líneas & $07.00-08.30 \mathrm{~h}$. & 20.00 h. y + \\
\hline Gran Canaria - Tenerife & 331 & 158 \\
\hline Tenerife - Gran Canaria & 403 & 144 \\
\hline Gran Canaria - Lanzarote & 216 & 72 \\
\hline Lanzarote - Gran Canaria & 216 & 173 \\
\hline Tenerife - La Palma & 216 & 86 \\
\hline La Palma - Tenerife & 144 & 158 \\
\hline Gran Canaria - Fuerteventura & 216 & 158 \\
\hline Fuerteventura - Gran Canaria & 216 & 173 \\
\hline Tenerife - Lanzarote & 144 & 29 \\
\hline Lanzarote - Tenerife & 72 & 101 \\
\hline Tenerife - Fuerteventura & 72 & 14 \\
\hline Fuerteventura - Tenerife & 72 & 86 \\
\hline Tenerife - El Hierro & 72 & 0 \\
\hline El Hierro - Tenerife & 0 & 0 \\
\hline Gran Canaria - La Palma & 72 & 14 \\
\hline La Palma - Gran Canaria & 0 & 72 \\
\hline Total & 2462 & 1438 \\
\hline
\end{tabular}

Fuente: elaboración propia a partir de los departamentos comerciales de Binter Canarias y Canaryfly (2020)

Se deduce pues que la situación es netamente distinta, pues si en las cuatro primeras rutas se ofertaban casi 3.300 plazas diarias de ida y vuelta en esas horas de mayor demanda, en otras no existía en ambos sentidos ninguna plaza en esas horas, como entre Tenerife y La Gomera, mientras que, en otros casos, en alguno de los sentidos tampoco se cumplía este requisito, 
como entre Tenerife y El Hierro. Como regla general, las conexiones entre la isla central y la periférica de la provincia adyacente, por ejemplo Tenerife-Fuerteventura o Gran Canaria-La Palma, además de las existentes con las islas con menor peso demográfico dentro de la misma provincia (La Gomera y El Hierro), se ven penalizadas por esta falta de accesibilidad en las franjas horarias de mayor relevancia para así disponer de mayor tiempo disponible en el destino. Como decimos, el análisis del tiempo disponible en destino supone uno de los principales objetivos de este trabajo. En este sentido, lejos de los planteamientos de algunos autores que sugieren que el tiempo de transporte puede llegar a ser una experiencia placentera (Jain, 2008), a nuestro juicio, el valor del tiempo, sobre todo en viajeros commuters en Canarias, se convierte en un factor negativo, incluso con más peso que las tarifas, en línea con muchos autores (Hernández Luis, 2000; Chowdhury, 2015; Antunes, 2018). En efecto, el valor del tiempo se ha plasmado en una mayor exigencia de rapidez en el transporte, a la vez que adecuación de los horarios de apertura de los lugares funcionales a este (Harris, 2004), aunque con claras connotaciones, pues la navegación marítima en fast ferries o la demanda de más frecuencias aéreas en aras de disminuir los tiempos de permanencia en los destinos, se traduce en una clara dicotomía entre el impacto económico positivo y el negativo por lo que afecta al medioambiente. Pero si partimos del beneficio económico, y considerando la metodología expuesta, se llega a la conclusión de que la disponibilidad de tiempo de los viajeros para resolver asuntos de carácter comercial o administrativo entre las dos islas centrales utilizando el modo aéreo, se puede considerar aceptable, ya que alcanza el 100 por cien del máximo de tiempo disponible en el que estos lugares funcionales se encuentran abiertos al público. Por su parte, en las rutas Gran Canaria-Lanzarote o Tenerife-La Palma, los porcentajes están muy próximos al 100 por cien, pues en estas existen aeronaves que pernoctan en la isla menor, facilitando la salida más temprana desde allí y contribuyendo con ello a evitar las deficiencias de disponibilidad horaria en el destino que si concurren en otras rutas. 
Tabla 10. Accesibilidad horaria a las "capitales" de otras islas de Canarias en viaje aéreo de un commuter en una jornada laboral (enero de 2020)

\begin{tabular}{|c|c|c|c|c|c|c|c|}
\hline \multirow[b]{2}{*}{ Rutas } & \multicolumn{2}{|c|}{$\begin{array}{l}\text { Bloques de } \\
\text { tiempo de ida }\end{array}$} & \multicolumn{3}{|c|}{ Bloques de tiempo en la vuelta } & \multicolumn{2}{|c|}{$\begin{array}{c}\text { Tiempo disponible en } \\
\text { lugares funcionales } \\
\text { abiertos (\%) }\end{array}$} \\
\hline & $\begin{array}{l}\text { Llega } \\
\text { avión }\end{array}$ & $\begin{array}{l}\text { Llega } \\
\text { capital }\end{array}$ & $\begin{array}{l}\text { Salida } \\
\text { capital }\end{array}$ & $\begin{array}{l}\text { Llegada al } \\
\text { embarque }\end{array}$ & $\begin{array}{l}\text { Salida } \\
\text { avión }\end{array}$ & Admon. & Comercial \\
\hline Tenerife-El Hierro & 08.35 & 08.50 & 17.00 & 17.15 & 17.55 & 88.10 & 62.50 \\
\hline El Hierro-Tenerife & 09.35 & 10.05 & 15.35 & 16.05 & 16.45 & 70.24 & 36.46 \\
\hline Tenerife-La Palma & 08.00 & 08.15 & 19.20 & 19.35. & 20.15 & 96.43 & 91.67 \\
\hline La Palma-Tenerife & 08.30 & 09.00 & 18.50 & 19.20 & 20.00 & 85.71 & 85.42 \\
\hline Tenerife-La Gomera & 10.00 & 10.45 & 16.25 & 17.10 & 17.50 & 60.71 & 33.33 \\
\hline La Gomera-Tenerife & 11.00 & 11.30 & 15.50 & 16.20 & 17.00 & 50.00 & 18.75 \\
\hline Tenerife-Gran Canaria & 07.30 & 08.00 & 19.50 & 20.20 & 21.00 & 100.00 & 97.92 \\
\hline Gran Canaria-Tenerife & 07.30 & 08.00 & 19.50 & 20.20 & 21.00 & 100.00 & 97.92 \\
\hline Tenerife-Fuerteventura & 08.00 & 08.15 & 19.35 & 19.50 & 20.30 & 96.43 & 91.67 \\
\hline Fuerteventura-Tenerife & 09.20 & 09.50 & 18.00 & 18.30 & 19.10 & 73.81 & 64.58 \\
\hline Tenerife-Lanzarote & 07.50 & 08.05 & 19.35 & 19.50 & 20.30 & 98.81 & 94.79 \\
\hline Lanzarote-Tenerife & 09.15 & 09.45 & 18.00 & 18.30 & 19.10 & 89.29 & 75.00 \\
\hline Gran Canaria-La Palma & 08.10 & 08.25 & 20.20 & 20.35 & 21.15 & 94.05 & 100.00 \\
\hline La Palma-Gran Canaria & 09.30 & 10.00 & 18.45 & 19.15 & 19.55 & 71.43 & 71.88 \\
\hline Gran Canaria-Fuerteventura & 07.40 & 07.55 & 20.35 & 20.50 & 21.30 & 100.00 & 100.00 \\
\hline Fuerteventura-Gran Canaria & 07.40 & 08.10 & 19.10 & 19.40 & 20.20 & 97.92 & 86.46 \\
\hline Gran Canaria-Lanzarote & 07.40 & 07.55 & 21.20 & 21.35 & 22.15 & 100.00 & 100.00 \\
\hline Lanzarote-Gran Canaria & 07.45 & 08.15 & 20.00 & 20.15 & 20.55 & 96.43 & 100.00 \\
\hline
\end{tabular}

Fuente: elaboración propia a partir de los horarios de Binter Canarias y Canaryfly (2020)

Los casos más deficitarios los encontramos en las líneas que unen las islas de El Hierro y La Gomera, donde la disponibilidad horaria se reduce incluso a menos del $20 \%$, por ejemplo cuando un residente de La Gomera se desplaza a Tenerife por motivos comerciales, siendo de menos del $40 \%$, es decir, de menos de tres horas en destino para asuntos comerciales cuando un residente en El Hierro se desplaza a Tenerife. Pero como decíamos, el desplazamiento desde las islas periféricas a las centrales siempre se ve desfavorecido, por lo que la accesibilidad en términos de tiempo, es un hándicap que compromete seriamente la cohesión territorial de las Islas.

Como ejemplo de todo ello mostramos en las Figuras 4 y 5, dos cartografías de isócronas de 30 minutos donde se exponen los tiempos reales de transporte desde y hacia San Sebastián de La Gomera, teniendo como origen o destino las capitales de Gran Canaria y Tenerife. Como se ha indicado, desde el año 2010, no existe línea aérea directa entre Gran Canaria y La Gomera, y 
todas las unidades de tráfico han de canalizarse por el nodo de Tenerife Norte. Ello ha motivado que si bien los ciudadanos de las capitales de ambas islas capitalinas arriben a San Sebastián de La Gomera a las 10,50 horas aproximadamente, en el sentido inverso estos tiempos se retrasan hasta las 11,30 hasta Santa Cruz de Tenerife y una hora más tarde para alcanzar Las Palmas de Gran Canaria, con la consiguiente vulnerabilidad de los viajeros de La Gomera al disponer de menos tiempo en las islas capitalinas.

Figura 4. Isócronas en viaje aéreo desde San Sebastián de La Gomera a las capitales insulares de Tenerife y Gran Canaria en enero de 2020

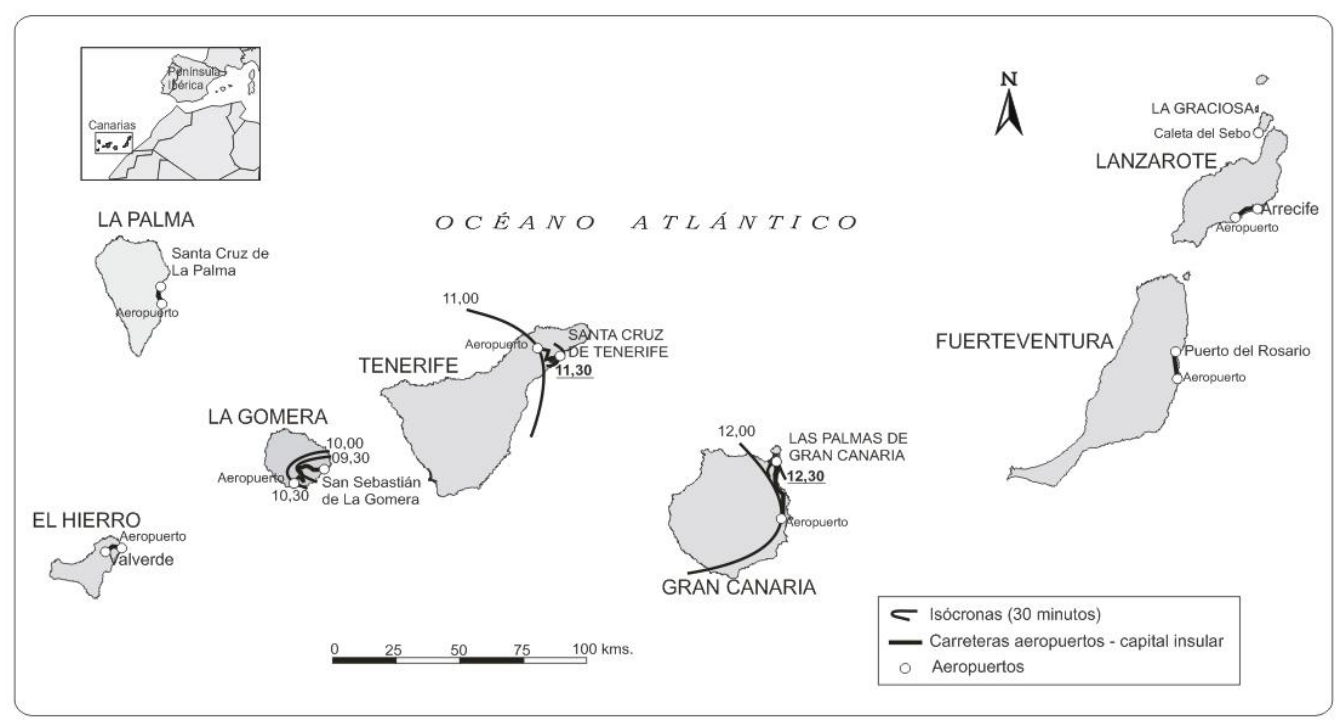

Fuente: elaboración propia a partir de los horarios de Binter Canarias (2020)

Figura 5. Isócronas en viaje aéreo desde las capitales insulares de Tenerife y Gran Canaria a San Sebastián de La Gomera en enero de 2020

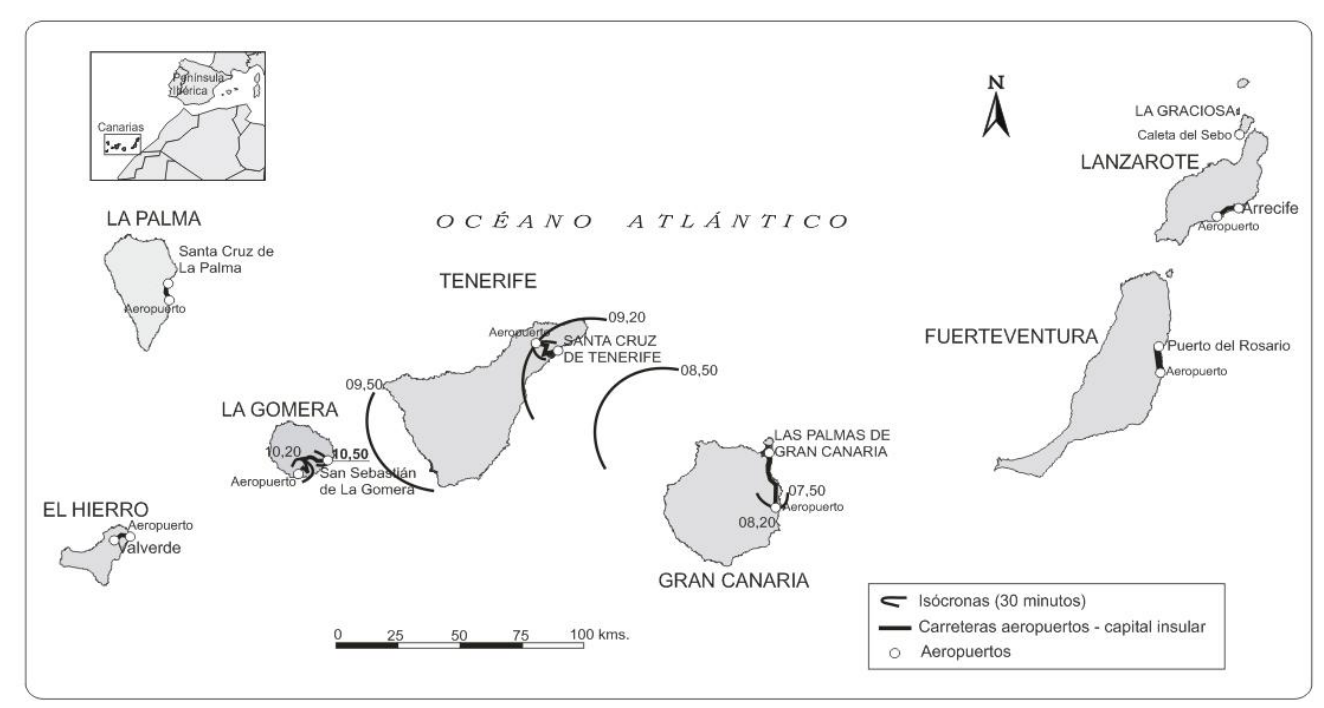

Fuente: elaboración propia a partir de los horarios de Binter Canarias (2020) 
El resultado es que esta vulnerabilidad por falta de disponibilidad de tiempo en los destinos y que se manifiesta por ejemplo en un desplazamiento aéreo por motivos administrativos, tiene un coste imputable a los salarios de cada categoría laboral y que se viene a añadir a las tarifas aéreas y terrestres. Así, toda llegada a la capital de destino con posterioridad a las 08.00 horas - o salida cuando se está en el destino antes de las 15.00-, representa un potencial coste para los viajeros. Por todo ello y porque las conexiones entre las dos islas centrales permiten una llegada al destino final antes de la citada franja horaria, el coste global del desplazamiento termina siendo notablemente inferior en relación con las conexiones del resto de la red, por ejemplo casi la mitad si se compara con el residente en La Gomera que se desplaza en avión a la capital de Tenerife (véase Tabla 11).

Desde un punto de vista evolutivo, tomando 21 años como referencia y según las programaciones de invierno de los operadores aéreos, el principal problema radica en las conexiones con las islas de El Hierro y de La Gomera, incluso con retrocesos significativos, pues el cambio de aeronave entre Gran Canaria y El Hierro en 2008, utilizando desde entonces un ATR de 72 plazas, en lugar del Beechcraft de 19, implicó pasar de dos conexiones diarias en cada sentido a una sola. Más grave aún fue lo sucedido en 2010 entre Gran Canaria y La Gomera, pues el citado cambio de aeronave supuso el cese de la línea directa y el tránsito de todos esos viajeros a través de Tenerife con los consiguientes costes económicos y de tiempo.

Como decíamos, si tomamos la ruta entre las dos islas centrales, la disponibilidad de tiempo, tanto comercial como administrativa, ha sido bastante alta y por tanto muy aceptable durante el período analizado. Incluso desde 2009 mejora ostensiblemente y en 2014 la situación se corresponde con la ideal, pues en los cuatro parámetros analizados se alcanza el 100 por cien de cobertura de los horarios administrativo y comercial (véase Figura 6). 
Tabla 11. Tarifa aérea y coste del tiempo de trabajo en un trayecto de ida, según las categorías laborales de mayor demanda de movilidad interinsular en rutas directas, imputable a la resolución de asuntos administrativos en las capitales insulares de destino en enero de 2020 (en euros)*

\begin{tabular}{|c|c|c|c|c|c|c|c|c|c|}
\hline \multirow[t]{2}{*}{ Líneas } & \multirow{2}{*}{$\begin{array}{l}\text { Tarifa } \\
\text { aérea y } \\
\text { terrestre }\end{array}$} & \multicolumn{2}{|c|}{$\begin{array}{c}\text { Directores y } \\
\text { gerentes }\end{array}$} & \multicolumn{2}{|c|}{$\begin{array}{l}\text { Profesionales } \\
\text { científicos e } \\
\text { intelectuales }\end{array}$} & \multicolumn{2}{|c|}{$\begin{array}{c}\text { Técnicos } \\
\text { profesionales } \\
\text { de apoyo }\end{array}$} & \multicolumn{2}{|c|}{$\begin{array}{c}\text { Todas las } \\
\text { categorías* * }\end{array}$} \\
\hline & & $\begin{array}{l}\text { Coste } \\
\text { tiempo }\end{array}$ & $\begin{array}{l}\text { Coste } \\
\text { total }\end{array}$ & $\begin{array}{l}\text { Coste } \\
\text { tiempo }\end{array}$ & $\begin{array}{l}\text { Coste } \\
\text { total }\end{array}$ & $\begin{array}{l}\text { Coste } \\
\text { tiempo }\end{array}$ & $\begin{array}{c}\text { Coste } \\
\text { total }\end{array}$ & $\begin{array}{l}\text { Coste } \\
\text { tiempo }\end{array}$ & $\begin{array}{l}\text { Coste } \\
\text { total }\end{array}$ \\
\hline Gran Canaria - Tenerife & 67,68 & 0,00 & 67,68 & 0,00 & 67,68 & 0,00 & 67,68 & 0,00 & 67,68 \\
\hline Tenerife - Gran Canaria & 67,68 & 0,00 & 67,68 & 0,00 & 67,68 & 0,00 & 67,68 & 0,00 & 67,68 \\
\hline Tenerife - La Gomera & 42,41 & 65,70 & 108,11 & 48,70 & 91,11 & 32,37 & 74,78 & 28,13 & 70,54 \\
\hline La Gomera - Tenerife & 42,41 & 83,62 & 126,03 & 61,99 & 104,40 & 41,20 & 83,61 & 35,81 & 78,22 \\
\hline $\begin{array}{l}\text { Gran Canaria - } \\
\text { Fuerteventura }\end{array}$ & 73,18 & 0,00 & 73,18 & 0,00 & 73,18 & 0,00 & 73,18 & 0,00 & 73,18 \\
\hline $\begin{array}{l}\text { Fuerteventura - Gran } \\
\text { Canaria }\end{array}$ & 73,18 & 29,86 & 103,04 & 22,13 & 95,31 & 14,71 & 87,89 & 12,79 & 85,97 \\
\hline Tenerife - La Palma & 53,16 & 5,97 & 59,13 & 4,43 & 57,59 & 2,94 & 56,10 & 2,56 & 55,72 \\
\hline La Palma - Tenerife & 53,16 & 23,89 & 77,05 & 17,71 & 70,87 & 11,77 & 64,93 & 10,23 & 63,39 \\
\hline $\begin{array}{l}\text { Gran Canaria - } \\
\text { Lanzarote }\end{array}$ & 65,93 & 0,00 & 65,93 & 0,00 & 65,93 & 0,00 & 65,93 & 0,00 & 65,93 \\
\hline $\begin{array}{l}\text { Lanzarote - Gran } \\
\text { Canaria }\end{array}$ & 65,93 & 5,97 & 71,90 & 4,43 & 70,36 & 2,94 & 68,87 & 2,56 & 68,49 \\
\hline Tenerife - Lanzarote & 59,16 & 1,99 & 61,15 & 1,48 & 60,64 & 0,98 & 60,14 & 0,85 & 60,01 \\
\hline Lanzarote - Tenerife & 59,16 & 41,80 & 100,96 & 30,99 & 90,15 & 20,60 & 79,76 & 17,90 & 77,06 \\
\hline Tenerife - El Hierro & 55,41 & 19,91 & 75,32 & 14,76 & 70,17 & 9,81 & 65,22 & 8,53 & 63,94 \\
\hline El Hierro - Tenerife & 55,41 & 49,77 & 105,18 & 36,90 & 92,31 & 24,52 & 79,93 & 21,31 & 76,72 \\
\hline Tenerife - Fuerteventura & 62,91 & 5,97 & 68,88 & 4,43 & 67,34 & 2,94 & 65,85 & 2,56 & 65,47 \\
\hline Fuerteventura - Tenerife & 62,91 & 43,80 & 106,71 & 32,47 & 95,38 & 21,58 & 84,49 & 18,76 & 81,67 \\
\hline Gran Canaria - La Palma & 74,93 & 9,95 & 84,88 & 7,38 & 82,31 & 4,90 & 79,83 & 4,26 & 79,19 \\
\hline La Palma - Gran Canaria & 74,93 & 47,78 & 122,71 & 35,42 & 110,35 & 23,54 & 98,47 & 20,46 & 95,39 \\
\hline
\end{tabular}

* Los datos para Canarias de ganancia por hora normal de trabajo se refieren a 2018.

** Se incluyen las restantes siete categorías laborales, totalizando diez.

*** Coste de la tarifa aérea puerta - puerta en taxi, excepto en La Gomera que se considera el transporte público. El coste del tiempo se ha obtenido en función de la ganancia media por hora normal de trabajo en Canarias, que fue de 10,23 euros en 2018, siendo de 23,89; 17,71; y 11,77 euros en el primero, segundo y tercer grupo de ocupación reseñados en esta tabla.

Fuente: elaboración propia a partir de la Encuesta Anual de Estructura Salarial (2020); Binter Canarias y Canaryfly 
Figura 6. Disponibilidad de tiempo en día laboral y en horario administrativo y comercial en las capitales insulares de Tenerife y Gran Canaria en viaje aéreo entre 2000 y 2020 (en \%)

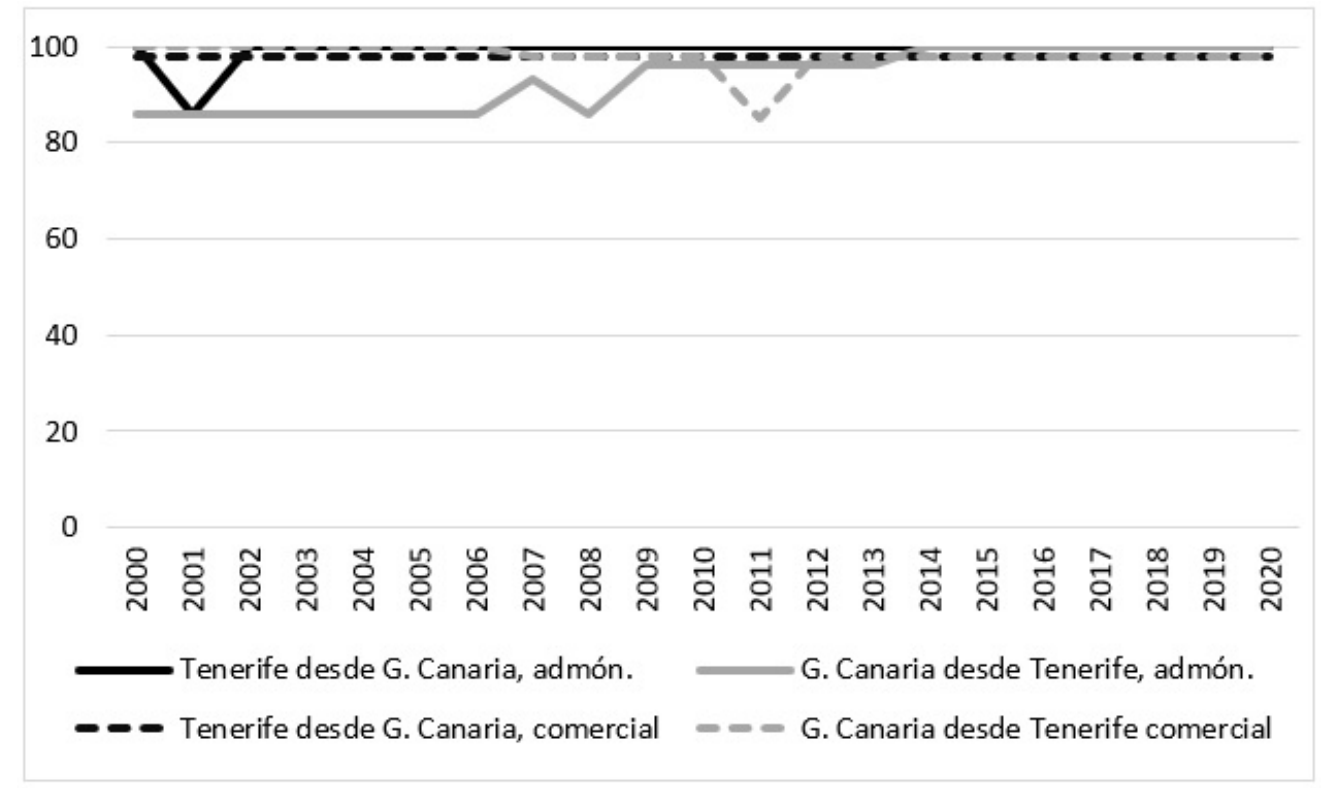

Fuente: elaboración propia a partir de los horarios de los operadores aéreos

En las rutas radiales dentro de la misma provincia el contexto es muy dispar, pues si dentro del conjunto oriental la situación se termina por asemejar al escenario óptimo entre las dos islas centrales, con valores entre el 90 y el 100 por cien de cobertura en los últimos años, en las occidentales, salvo la conexión entre La Palma y Tenerife con mejores resultados, los porcentajes se muestran deficientes, con un mínimo de solo un $20 \%$ de disponibilidad horaria comercial en Santa Cruz de Tenerife para el residente en La Gomera. Además, dentro de estas últimas rutas, se favorece el sentido del pasajero central que se desplaza a la isla periférica, estadísticamente con mayor capacidad adquisitiva para afrontar una hipotética pernoctación en el destino debido a la deficiencia horaria de la operativa aérea, a la vez que también menos necesitado de desplazamientos interinsulares con carácter recurrente, por lo que el resultado no puede ser más perverso para el viajero periférico. 
Figura 7. Disponibilidad de tiempo en día laboral y en horario administrativo y comercial en las capitales insulares entre las islas centrales y sus islas de la provincia en viaje aéreo entre 2000 y 2020 (en \%)
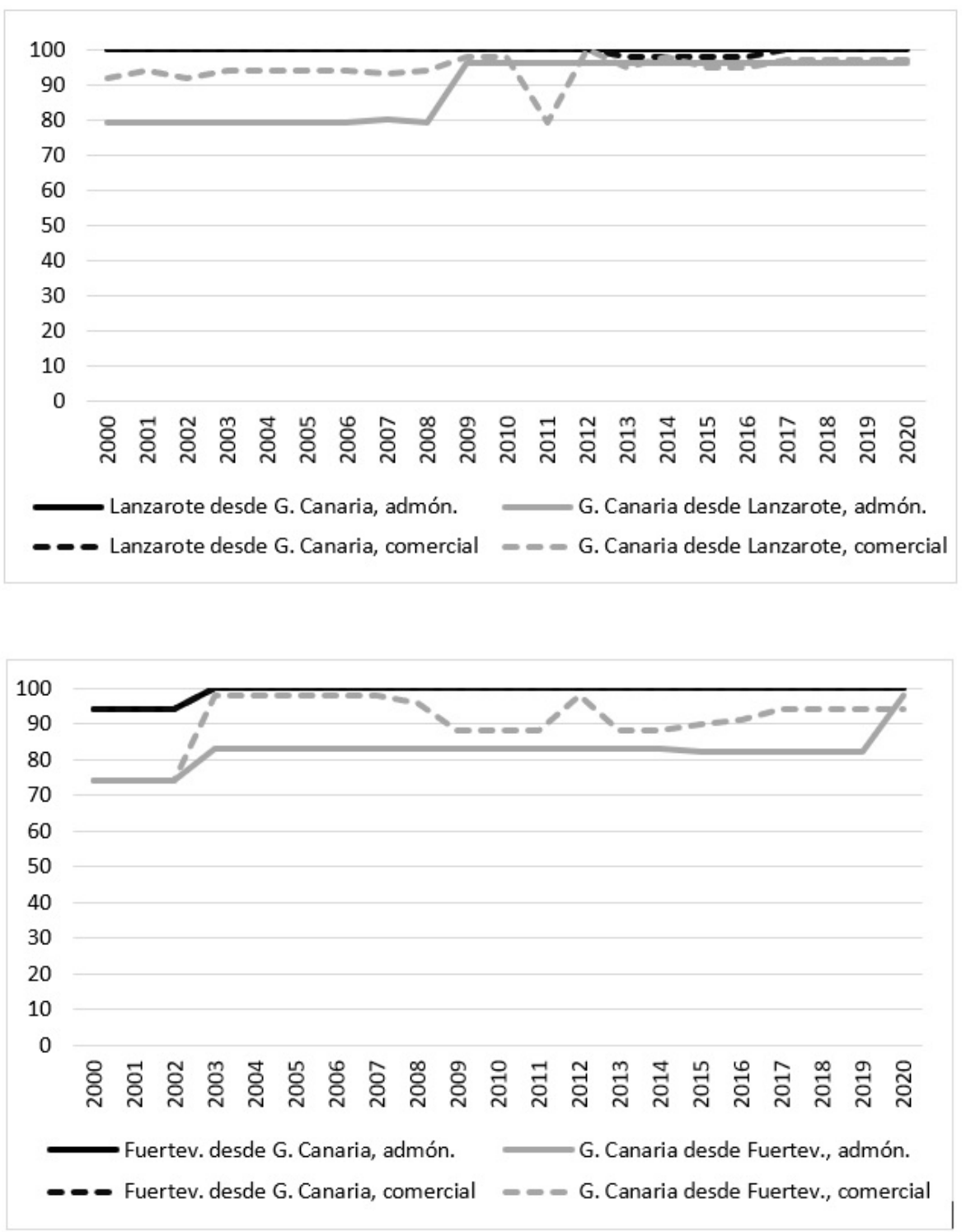
Figura 7. Continuación
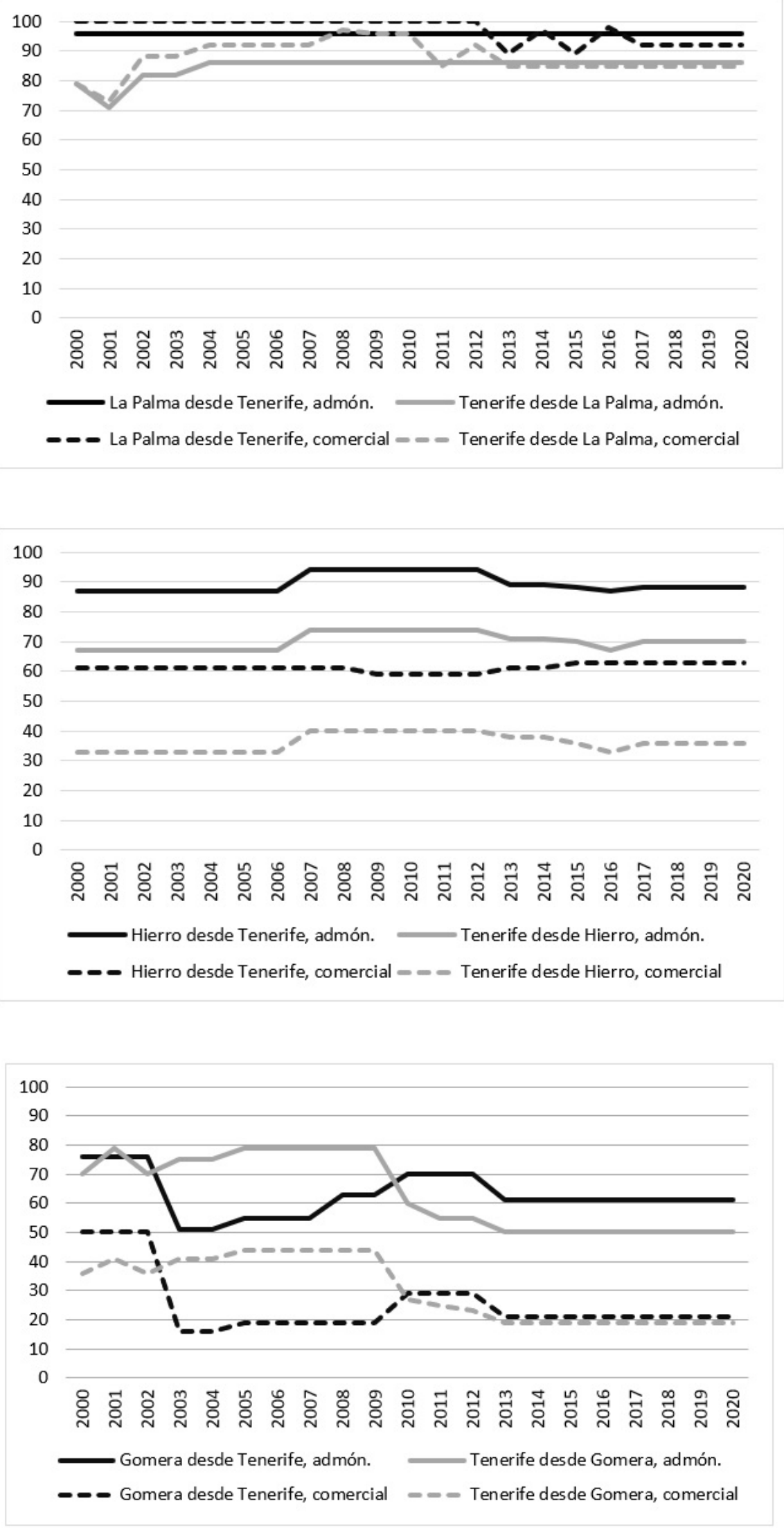

Fuente: elaboración propia a partir de los horarios de los operadores aéreos 
Por lo que respecta a las rutas interprovinciales la situación también es bastante dispar. Así, mientras las conexiones horarias han mejorado ostensiblemente con Lanzarote y Fuerteventura desde Tenerife, pues superaban disponibilidades de tiempo superiores al $90 \%$ para los viajeros desde la isla central, aunque rondasen el 70 \% en sentido inverso, desde Gran Canaria hacia las tres islas occidentales el panorama ha sido muy deficitario, salvo la conexión con La Palma, particularmente desde el año 2003. Como decimos, las rutas entre Gran Canaria y las islas de El Hierro y La Gomera son las que peores datos de conectividad horaria round trip ofrecían de la serie analizada, pues solo cuando existieron dos frecuencias diarias en cada sentido, fue posible regresar en la misma jornada. Así pues, los períodos 2005-2007 entre Gran Canaria y El Hierro y 2003-2009, entre Gran Canaria y La Gomera, fueron los únicos que arrojaron datos. En particular aquí se demostró la escasez de tiempo en el destino, sobre todo cuando se consideró la disponibilidad comercial, afectada por unos horarios aéreos más tempranos en horas de la tarde, siendo paradigmático que los residentes en La Gomera solo dispusieran de un $19 \%$ del tiempo comercial en Gran Canaria, con una probabilidad muy alta, por tanto, de pernoctación en el destino con el consiguiente coste económico y de tiempo que ello conllevaba.

Figura 8. Disponibilidad de tiempo en día laboral y en horario administrativo y comercial en las capitales insulares desde las islas centrales a las periféricas de la otra provincia en viaje aéreo entre 2000 y 2020 (en \%)

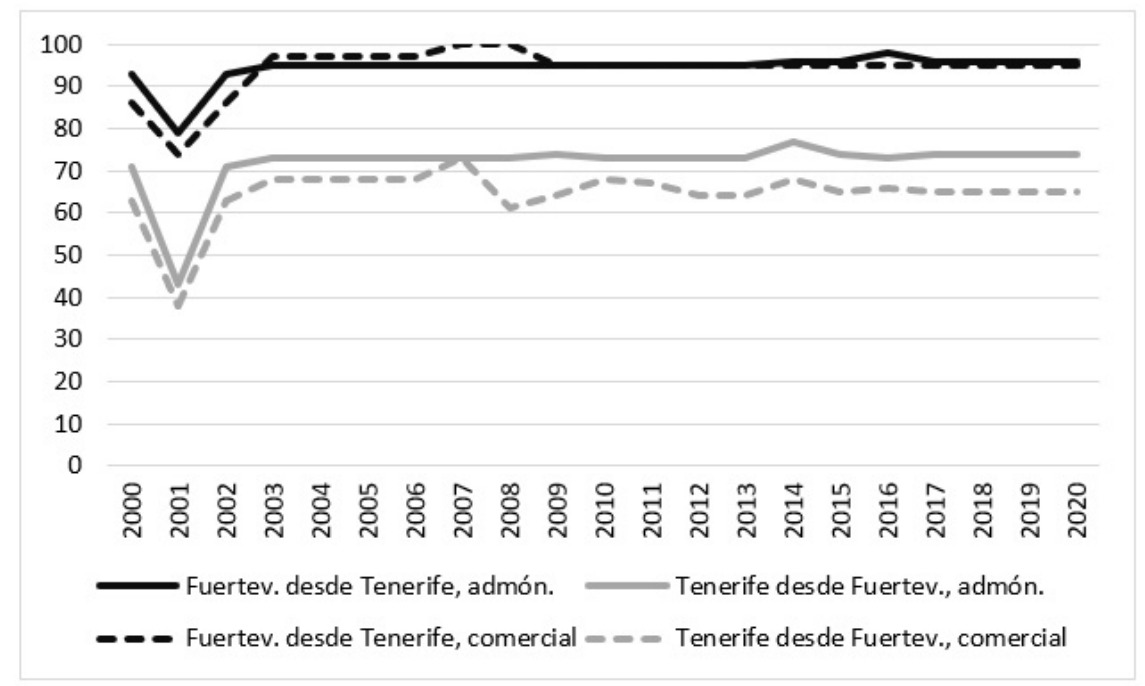


Figura 8. Continuación
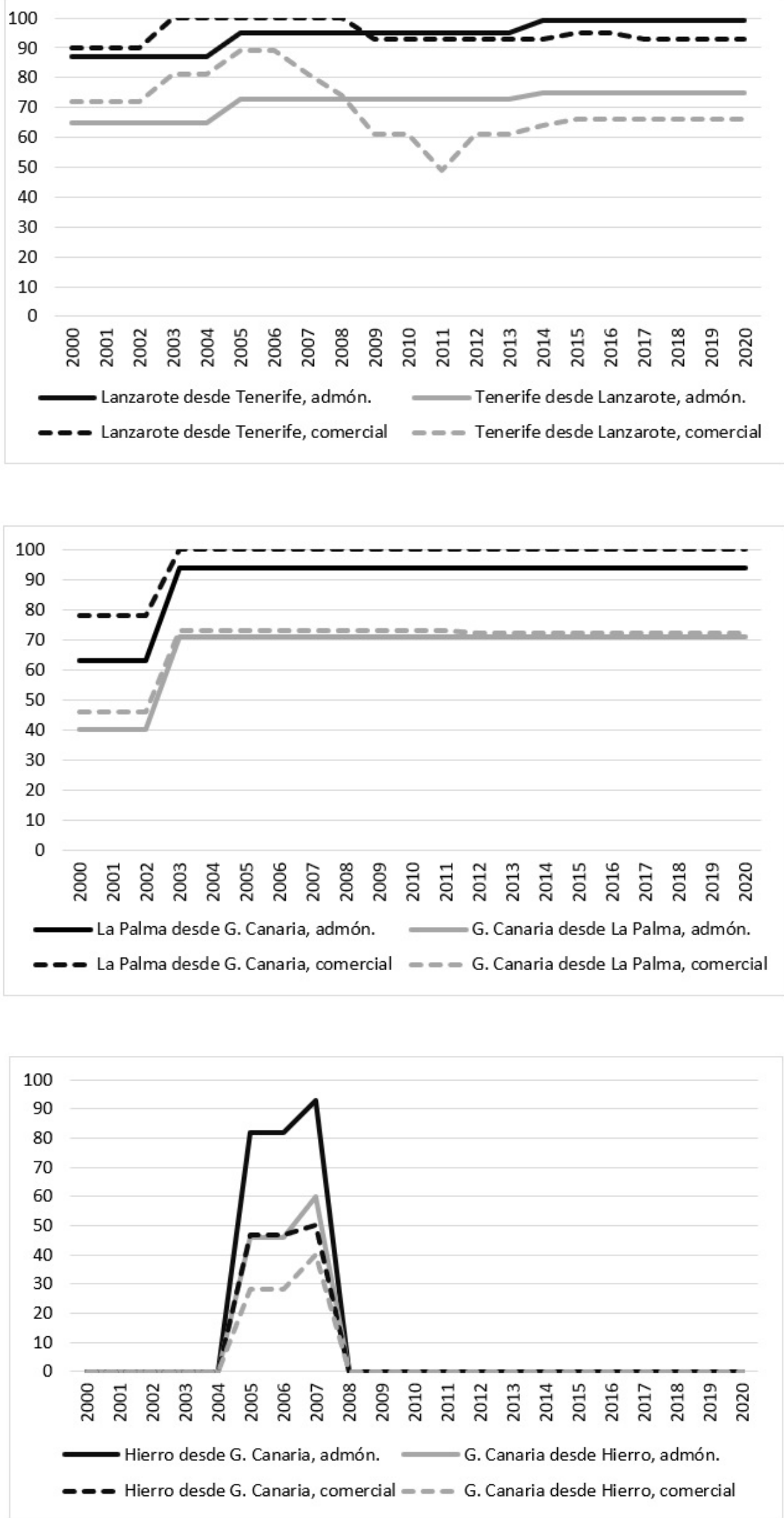
Figura 8. Continuación

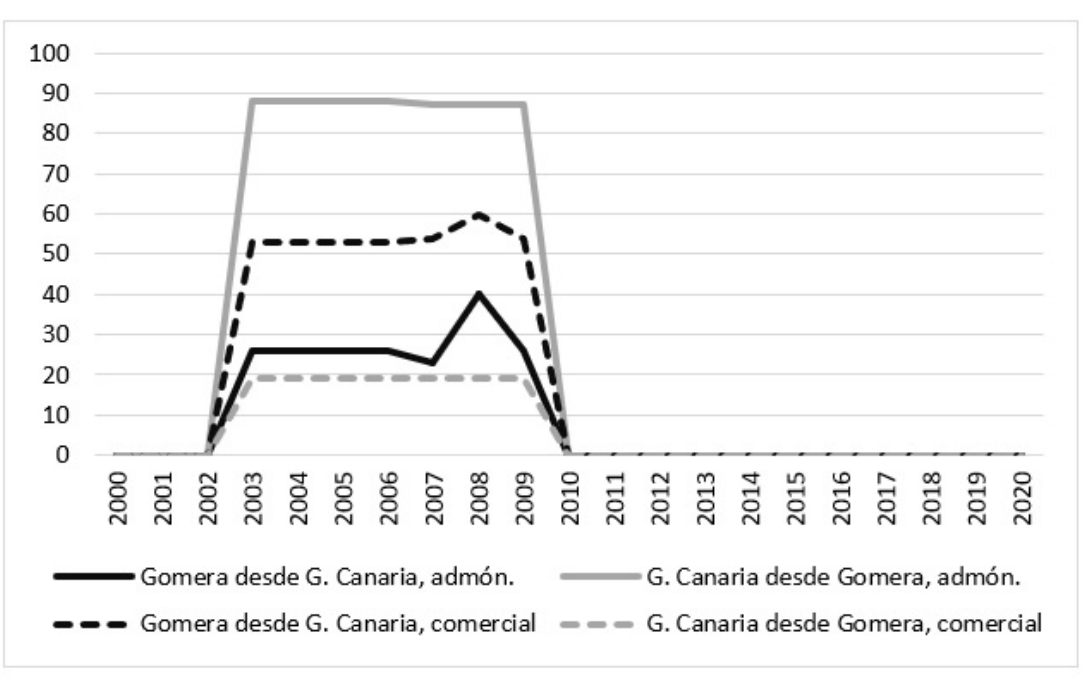

Fuente: elaboración propia a partir de los horarios de los operadores aéreos

\section{Resultados y discusión}

Algunos indicadores nos acercan a la necesidad de la mejora de la accesibilidad aérea interinsular en Canarias, como por ejemplo que los trabajadores del $25 \%$ de las empresas de las Islas se ven obligados a realizar desplazamientos interinsulares, lo que representa unos 40 mil desplazamientos mensuales. Casi el $65 \%$ de estos desplazamientos lo es en avión. Tan solo el sobrecoste por el incremento de la duración de los viajes (pérdida de tiempo, alojamientos y manutención), debido gran parte de las veces a una deficitaria accesibilidad, supone de media a las empresas más de 15 mil euros al año, imputable en el $82 \%$ de los casos a la pérdida de tiempo (UTE Eco-CoRe, 2019). A ello hay que unirle los viajes particulares por motivos sanitarios, comerciales, etc., que también demandan una óptima accesibilidad, sobre todo round trip, en aras de evitar el sobrecoste de la pernoctación en destino. A su vez, los commuters interinsulares representan la elevada cifra del $48 \%$ de los pasajeros entre Tenerife y Gran Canaria, mientras descienden al $27 \%$ con las restantes islas (Gobierno de Canarias, 2010), dato este último, en relación con el primero, que podría estar demostrando una demanda insatisfecha.

Los datos aportados en el epígrafe sobre la accesibilidad aérea interinsular demuestran una absoluta concordancia con estos informes que, sin analizar en profundidad el problema de la accesibilidad, solo constatan el elevado déficit económico y de tiempo que genera la ausencia de una buena conexión, sobre todo con las islas periféricas. Por ello, extraemos varios puntos para la discusión: 
1. La conexión aérea es estratégica en un medio insular. Además, en Canarias las islas periféricas carecen de muchos servicios y, por ello, la dependencia con respecto a las centrales es muy elevada, hasta el punto de que los residentes beneficiarios de la bonificación aérea alcanzan anualmente hasta casi el 80 y 65 \% de la población de las islas de El Hierro y La Palma respectivamente, frente a solo el $15 \%$ de los residentes en las islas centrales (Ramos Pérez, 2020). El análisis de estas cifras requiere de una especial atención a estas conexiones con las islas periféricas y no solo entre las centrales. Es más, sobre todo debería facilitarse una mejor accesibilidad en los sentidos de línea isla periférica-central, dado que gran parte de los commuters, en relación con su población, nos lo encontramos en estas islas, además con menor renta para afrontar los viajes.

2. Las tarifas aéreas para un residente, no puerta - puerta como se considera en este estudio, sino solamente el servicio aéreo, han mejorado ostensiblemente en los últimos años, hasta el punto de que se detectan algunos comportamientos no deseables de presunto exceso de viajes que, indudablemente repercuten en las arcas públicas (Ramos Pérez, 2020). Desde 2017 en que la subvención al residente interinsular pasó en Canarias, al igual que en Baleares, desde el 50 al $75 \%$, el comportamiento de la demanda ha sido al alza, si exceptuamos el período de la pandemia. Indudablemente, para una ruta que permita con cierta holgura el round trip en la misma jornada, la intervención al alza en esta subvención es netamente beneficiosa para el residente, aunque como decimos, discutible para las arcas públicas e incluso el medio ambiente. Así pues, se deduce que las tarifas aéreas dejan de ser el obstáculo que fueron en el pasado y dejan paso sobre todo al problema de la disponibilidad de horarios.

3. La sistemática introducción de nuevos operadores aéreos en la red interinsular, sobre todo desde comienzos de Siglo - muchas veces por el abandono de otros-, ha supuesto una mayor oferta de aeronaves y asientos en determinadas horas punta, especialmente en las primeras y últimas horas del día, cubriendo los déficits de plazas que se registraban en estas franjas horarias (Hernández Luis, 2018). Por tanto, la carencia de plazas ha dejado de ser un grave problema, excepto fechas del año muy puntuales.

4. La conectividad interinsular ha mejorado sustancialmente a través del incremento de frecuencias, aunque se han perdido algunas conexiones de baja demanda, reemplazándose a través del hub de Tenerife Norte sobre todo, ya que el principal nodo afectado es La Palma 
que ha perdido sus conexiones directas con El Hierro, Lanzarote y Tenerife Sur. En especial, ello ha repercutido negativamente en la tarifa y el tiempo de viaje.

5. Llegados a este punto y en el actual contexto, parece demostrarse que los horarios aéreos son más importantes que las tarifas de los operadores, pues los horarios condicionan la disponibilidad de tiempo en destino de los viajeros. De este modo, el incremento del viaje en tan solo un día, ya implica mayores costes económicos que la tarifa aérea en si para un residente que ha accedido a la bonificación como tal. Así, para un residente en La Gomera que se desplace a Tenerife, con tan solo 210 minutos disponibles en la capital de la isla central para solucionar asuntos administrativos (solo 90 minutos en horario comercial), el riesgo de una pernoctación ya comienza a ser elevado. Y aquí caben dos posibilidades, la primera y evidentemente la mejor -aunque menos posible ya que se sigue necesitando disponibilidad de tiempo en la mañana siguiente-, es que el viajero opte por regresar el día siguiente en la primera frecuencia, aunque no alcanzaría la capital de La Gomera hasta las 10,45 horas. Para el caso de un director o gerente y de acuerdo a su categoría laboral, tan solo ese tiempo de 2,45 horas del día siguiente se correspondería con casi 54 euros de tiempo laboral perdido en La Gomera, sin añadirle aún el más variable coste del alojamiento y de la manutención de la tarde-noche anterior. Si como es más viable, el viajero regresa en horas de la tarde, entonces la tarifa aérea y terrestre termina por ser anecdótica frente a estos otros costes. Cabría pues plantear una mejora ostensible de los horarios de operatividad aérea, especialmente con las islas de El Hierro y La Gomera, en aras de favorecer una mayor integración territorial de las Islas como preconiza el denominado Eje Transinsular de Transportes.

6. Dentro del cómputo de los horarios, cabe introducir el problema de los accesos terrestres a las capitales insulares de las islas centrales, muy congestionadas por lo general hasta las 9.00 horas aproximadamente. Éste es un problema añadido que sufre el pasajero periférico y que el de la isla central no va a soportar en las otras islas. Por tanto, se deduce que es más urgente la primera operatividad horaria del día en el sentido isla periférica-isla central, al menos dentro de las islas de la misma provincia, con mayor demanda y no en el sentido inverso, tal y como se ha venido operando con las islas más afectadas de El Hierro y La Gomera. Este es un sistema que se ha ido implantando con éxito para los sentidos de línea La Palma-Tenerife (2004); Lanzarote-Gran Canaria (2009); y Fuerteventura-Gran Canaria (2020), pero que aun así no cumple con la máxima de que la operatividad desde la isla periférica anteceda incluso su salida en relación con el sentido isla central - isla periférica, 
partiendo de los mencionados problemas de acceso terrestre en las islas centrales. Desde luego la apertura más temprana y cierre más tardío de las infraestructuras aeroportuarias, sobre todo de las islas periféricas occidentales -y acompañado de una política de los operadores en este sentido para cubrir dichas franjas horarias-, no debe ser un escollo para la resolución de este grave problema de accesibilidad aérea en Canarias.

7. Parece pertinente que la solución a esta desigualdad debiera centrarse en la mejora de la planificación horaria, partiendo de los horarios en que los comercios y las administraciones permanecen abiertos. A su vez, la operatividad con aeronaves de inferior capacidad en algunas rutas de menor densidad (las conexiones de Gran Canaria con El Hierro y La Gomera), posibilitarían al menos dos frecuencias diarias en cada sentido, siendo ésta la condición mínima exigible para el round trip en una misma jornada.

\section{Conclusiones}

El transporte aéreo en los medios insulares adquiere un carácter estratégico, máxime cuando su utilización, como en Canarias, presenta una alta cuota de round trip en la misma jornada. Es el único modo de transporte de pasajeros - salvo en travesías marítimas de escaso tiempo-, que consigue paliar la lejanía y fragmentación territorial. Ahora bien, la existencia per se de conexión directa no implica una accesibilidad idónea. Conscientes de ello, las obligaciones de servicio público aéreas de Canarias ya imponían en 2006 determinados parámetros de calidad (asientos, frecuencias, tarifas, exigencia de un mínimo de disponibilidad de tiempo en destino, etc.).

Como hemos demostrado y particularmente en las dos últimas décadas, se ha mejorado bastante la red aérea interinsular, pues se ha consolidado durante varios años una cierta competencia, sobre todo entre dos operadores, dando lugar a un esfuerzo de estas por adaptarse a la demanda. Fruto de ello es la constante renovación de la flota, el incremento de frecuencias con una mejor dispersión horaria durante la jornada, el consiguiente aumento de plazas disponibles, la moderación de tarifas, el incremento de la subvención al residente, la pernoctación en La Palma, Lanzarote y Fuerteventura de aeronaves para así facilitar una mayor disponibilidad de tiempo de los viajeros en Tenerife y Gran Canaria, la facturación electrónica reduciendo los tiempos en esta faceta, etc.

Han sido pues avances muy significativos en aras de la mentada integración territorial. Pero como preconizaban las obligaciones de servicio público, también con un cierto sesgo asegurando en mayor medida la conectividad de las islas de mayor peso demográfico, la 
exigencia de un mínimo de disponibilidad de tiempo en destino es quizá en la actualidad uno de los principales problemas de la red interinsular, sobre todo para los residentes en La Gomera y El Hierro y, especialmente, de los de esta última, ya que el transporte alternativo, el marítimo, no está tan desarrollado como en La Gomera. En efecto, los tiempos disponibles en las islas centrales de los habitantes de estas islas son francamente bajos y, por ende, la probabilidad de pernoctar en el destino se incrementa con todos sus costes derivados $y$, además, en un contexto de renta de estos ciudadanos inferior. A ello habría que añadirle la congestión de las autopistas en las islas centrales, que aún reduce en mayor grado -y en términos reales, aunque difícil de concretar con exactitud-, el tiempo disponible de los viajeros periféricos, ya que retrasa las llegadas a las capitales insulares y, además, en las horas vespertinas, cuando tiene lugar el regreso a sus islas de residencia, los viajeros tienden a abandonar antes estas urbes en previsión de los posibles efectos del tiempo extra de una potencial congestión viaria. Por ello decimos que esos teóricos 70 minutos necesarios en las islas centrales para el regreso desde la capital insular hasta la puerta de la aeronave (30 de carretera y 40 en facturación y embarque), podría ser algo mayor, hecho que reafirma aún más la necesidad de incrementar los tiempos disponibles de los viajeros periféricos en las islas centrales. Sin embargo, el viajero de las islas centrales no se va a encontrar con este problema en las islas periféricas, pues la congestión viaria en estas es prácticamente inexistente $y$, además, los tiempos de transporte terrestre son menores, si exceptuamos los pertinentes en La Gomera.

En resumen, se ha mejorado significativamente la red aérea interinsular, pero de manera desigual, ya que demostramos que, en la actualidad, la disponibilidad de tiempo es uno de los factores que más contribuye a la desintegración territorial de las Islas, en especial para los residentes en las islas periféricas.

Por último, habría que indicar la importancia de esta investigación, ya que es un instrumento de planificación para los operadores de transporte, al igual que sirve de soporte para que las administraciones públicas puedan demandar a los operadores unas determinadas condiciones mínimas del servicio sujetas a unos horarios con mayor dispersión entre la primera y última frecuencia, pero también con el compromiso de la administración de ampliar los horarios de operatividad de las infraestructuras aeroportuarias. 
Agradecimientos: Expreso mi mayor consideración y agradecimiento a las/os evaluadoras/es de este trabajo, especialmente por la dedicación de su tiempo y sus contribuciones y recomendaciones muy certeras para la mejora de esta publicación.

Declaración responsable: El autor declara que no existe ningún conflicto de interés relacionado con la publicación de este artículo. 


\section{Bibliografía}

Abásolo Alessón, I., García Pérez, L., Aguiar lbáñez, R., \& Amador Robayna, A. (2008). Análisis del efecto de la condición de "doble insularidad" sobre la equidad en la utilización de servicios sanitarios públicos: el caso de las Islas Canarias. Investigaciones Regionales, 13, 159175. https://old.aecr.org/images/ImatgesArticles/2008/066\%20ABASOLO.pdf

AIREF (2020). Infraestructuras de transporte. Madrid. Retrieved from https://www.airef.es/wpcontent/uploads/2020/09/INFRAESTRUCTURAS/200730.-INFRAESTRUCTURAS.-ESTUDIO.pdf

Antunes, A. P., Santos, M. G., Pita, J. P., \& Menezes, A. G. (2018). Study on the evolution of the air transport network of the Azores. Transportation Research Part A: Policy and Practice, 118, 837-851. https://doi.org/10.1016/j.tra.2018.10.021

Ashiru, O., Polak, J. W., \& Noland, R. B., (2003). Space-time user benefit and utility accessibility measures for individual activity schedules. Transportation Research Record: Journal of the Transportation Research Board, 1854(1), 62-73. https://doi.org/10.3141/1854-07

Barret, S. D. (1999). Peripheral market entry, product differentiation, supplier rents and sustainnability in the deregulated European aviation market - a case study. Journal of Air Transport Management, 5(1), 21-30. https://doi.org/10.1016/S0969-6997(98)00033-7

Benenson, I., Martens, K., Rofé, Y., \& Kwartler, A. (2011). Public transport versus private car GIS-based estimation of accessibility applied to the Tel Aviv metropolitan area. Annals of Regional Science, 47(3), 499-515. https://doi.org/10.1007/s00168-010-0392-6

Bocarejo, J. P., \& Oviedo, D. R. (2012). Transport accessibility and social inequities: a tool for identification of mobility needs and evaluation of transport investments. Journal of Transport Geography, 24, 142-154. https://doi.org/10.1016/j.jtrangeo.2011.12.004

Bowen, J. (2000). Airline hubs in Southeast Asia: national economic development and nodal accesibility. Journal of Transport Geography, 8(1), pp. 25-41. https://doi.org/10.1016/S09666923(99)00030-7

Brookfield, H. C. (1980). The transport factor in island development. In R. T. Shand (Ed.), The Island States of the Pacific and Indian Oceans: Anatomy of Development. Development Studies Centre Monograph, 23 (pp. 201-238). Canberra: Australian National University.

Butera, S., \& Ciaccio, G. (2002). Aspetti e tendenze dell'economia siciliana. Milano: II Mulino. 
Button, K. J., \& Taylor, S. (2000). International air transportation and economic development. Journal of Air Transport Management, 6(4), 209-222. https://doi.org/10.1016/S0969$\underline{6997(00) 00015-6}$

Calmet, D., \& Capurro, J. M. (2011). El tiempo es dinero. Cálculo del valor social del tiempo en Lima Metropolitana para usuarios de transporte urbano. Revista Estudios Económicos, 20, 7386. https://www.bcrp.gob.pe/docs/Publicaciones/Revista-Estudios-Economicos/20/ree-20calmet-capurro.pdf

Castanho, R. A., Naranjo Gómez, J. M., Vulevic, A., Behradfar, A., \& Couto, G. (2021). Assessing Transportation Patterns in the Azores Archipelago. Infrastructures, 6(10), 116. https://doi.org/10.3390/infrastructures6010010

Chang, J. S. (2010). Assessing travel time reliability in transport appraisal. Journal of Transport Geography, 18(3), 419-425. https://doi.org/10.1016/j.jtrangeo.2009.06.012

Chowdhury, S., Ceder, A., \& Schwalger, B. (2015). The effects of travel time and cost savings on commuters' decision to travel on public transport routes involving transfers. Journal of Transport Geography, 43, 151-159. https://doi.org/10.1016/j.jtrangeo.2015.01.009

Coccossis, H., \& Nijkamp, P. (1995). Overcoming isolation: information and transportation networks in development strategies for peripheral areas. Berlin: Springer.

Commission of the European Communities (2005). Trans-european transport network. Ten- $t$ priority axes and projects 2005. Luxemburgo: Office for Official Publications of the European Communities. Retrieved from

https://ec.europa.eu/ten/transport/projects/doc/2005_ten_t_en.pdf

Cross, M., \& Nutley, S. (1999). Insularity and Accessibility: the Small Island Communities of Western Ireland. Journal of Rural Studies, 15(3), 317-330. https://doi.org/10.1016/S0743$\underline{0167(98) 00062-X}$

Deidda, M. (2016). Insularity and economic development: a survey. International Review of Economics, 63, 107-128. https://doi.org/10.1007/s12232-015-0238-8

Durán Heras, Má. (2006). El valor del tiempo. ¿Cuántas horas te faltan al día? Madrid: Espasa-Calpe.

Durán-Hormazábal, E., \& Tirachini, A. (2016): Estimation of travel time variability for cars, buses, metro and door-to-door public transport trips in Santiago, Chile. Research in Transportation Economics, 59, 26-39. https://doi.org/10.1016/j.retrec.2016.06.002 
Ettema, D., \& Timmermans, H., (2007). Space-time accessibility under conditions of uncertain travel times: Theory and numerical simulations. Geographical Analysis, 39(2), 217240. https://doi.org/10.1111/j.1538-4632.2007.00702.x

Eurisles (1996). Systems of transport in the islands. París: L'Harmattan.

European Commission (2001). White Paper: European transport policy for 2010: time to decide. Luxemburg: Office for Official Publications of the European Communities. Retrieved from https://ec.europa.eu/transport/sites/transport/files/themes/strategies/doc/2001_white_ paper/lb_com_2001_0370_en.pdf

European Commission (2011). White Paper on transport. Luxemburg: Office for Official Publications of the European Communities. Retrieved from https://ec.europa.eu/transport/sites/transport/files/themes/strategies/doc/2011_white_ paper/white-paper-illustrated-brochure_en.pdf

Farrington, J. H. (2007). The new narrative of accessibility: its potential contribution to discourses in (transport) geography. Journal of Transport Geography, 15, 319330. https://doi.org/10.1016/j.jtrangeo.2006.11.007

Fundación Tomillo (2001). Los costes de ultraperiferia de la economía canaria. Santa Cruz de Tenerife: Consejería de Economía, Hacienda y Comercio. Retrieved from http://www.datosdelanzarote.com/uploads/doc/20060525132840418costes_ultraperifer ia-01.pdf

Fernández Tabales, A., Pedregal Mateos, B., Rodríguez Mateos, J. C., Pita Lopez, Ma, F., \& Zoido Naranjo, F. (2009). El concepto de cohesión territorial. Escalas de aplicación, sistemas de medición y políticas derivadas, Boletín de la Asociación de Geógrafos Españoles, 50, 157172. http://age.ieg.csic.es/boletin/50/06\%20FERNANDEZ.pdf

Gámir Orueta, A., \& Ramos Pérez, D. (2002). Transporte aéreo y territorio. Madrid: Ed. Ariel.

García Gordillo, J. L. (1983). Competencia y complementariedad del transporte aéreo y marítimo en Canarias. In El transporte regional en Canarias: III Jornadas de Estudios Económicos Canarios (pp. 223-234): Las Palmas de Gran Canaria.

Glorieux, I., Mestdag, I., \& Minnen, J. (2008). The coming of the 24-hour economy? Changing work schedules in Belgium between 1966 and 1999. Time \& Society, 17(1), 6383. https://doi.org/10.1177/0961463X07086310 
Gobierno de Canarias (1985). El transporte interinsular canario "transporte aéreo". Las Palmas de Gran Canaria: Consejería de Turismo y Transportes (Unpublished).

Gobierno de Canarias (1998). Libro Blanco de los transportes en Canarias. Las Palmas de Gran Canaria: Consejería de Turismo y Transportes.

Gobierno de Canarias (2010). Cuantificación de la diferencia en los costes derivados de la situación de ultraperiferia que soportan las empresas canarias respecto a sus homólogos continentales. Las Palmas de Gran Canaria: Consejería de Economía y Hacienda (Unpublished).

Graham, B. (1998). Liberalization, regional economic development and the geography of demand for air transport in the European Union, Journal of Transport Geography, 6(2), 87104. https://doi.org/10.1016/S0966-6923(98)00003-9

Graham, B., \& Guyer, C. (1999). Environmental sustainability, airport capacity and European air transport liberalization: irreconcilable goals?. Journal of Transport Geography, 7(3), 165180. https://doi.org/10.1016/S0966-6923(99)00005-8

Graham, A. (2000). Demand for leisure air travel and limits to growth. Journal of Air Transport Management, 6(2), 109-118. https://doi.org/10.1016/S0969-6997(99)00031-9

Grubesic, T. H., \& Fangwu, W. (2013). Essential Air Service: a local, geographic market perspective. Journal of Transport Geography, 30, 1725. https://doi.org/10.1016/j.jtrangeo.2013.02.008

Gwilliam, K. M. (1997). The value of time in economic evaluation of transport projects. The World Bank. Retrieved from

http://documents1.worldbank.org/curated/en/759371468153286766/pdf/816020BRIOInfrO0 Box379840B0OPUBLICO.pdf

Hägerstrand, T. (1970). What about people in regional science?. Papers of the Regional Science Association, 24(1), 6-21. https://doi.org/10.1007/BF01936872

Harris, P., Lewis, J., \& Adam, B., (2004). Time, sustainable transport and the politics of speed. World Transport Policy \& Practice, 10(2), 5-11. http://www.ecologica.co.uk/pdf/wtpp10.2.pdf

Hazledine, T., \& Collins, S. (2011). Paying the pilot? The economics of subsidising international air travel to small remote island nations with large diaspora. Journal of Air Transport Management, 17(3), 187-194. https://doi.org/10.1016/j.jairtraman.2010.12.008 
Hernández Luis, J. Á. (2000). Accesibilidad horaria en el transporte interinsular aéreo de Canarias. Revista de Estudios de Construcción y Transportes, n 89, Madrid: Ministerio de Fomento.

Hernández Luis, J. Á. (2002). Temporal accesibility in archipelagos: inter-island shipping in the Canary Islands. Journal of Transport Geography, 10, 231239. https://doi.org/10.1016/S0966-6923(02)00014-5

Hernández Luis, J. Á. (2003). El transporte exterior en La Palma. Santa Cruz de La Palma: Cabildo Insular de La Palma.

Hernández Luis, J. Á. (2004). The role of inter-island air transport in the Canary Islands. Journal of Transport Geography, 12(3), 235-244. https://doi.org/10.1016/j.jtrangeo.2004.04.005

Hernández Luis, J. Á. (2018). Diagnóstico del eje transinsular de transportes en el contexto de la integración territorial de Canarias. Boletín de la Asociación de Geógrafos Españoles, 77, 549580. https://doi.org/10.21138/bage.2551

Hoyle, B., \& Knowles, R. (Eds.) (1998). Modern Transport Geography. London: Wiley, 2nd Edition.

Hoyle, B. S. (1999): Islands, transport and development. In Biagini, E., \& Hoyle, B. S. (Eds.) Insularity and development: international perspectives on islands. London: Pinter.

Istac, (2020). Estadística de la Evolución Histórica de la Población. Gobierno de Canarias. Retrieved from http://www.gobiernodecanarias.org/istac/jaxiistac/menu. do? uripub=urn:uuid:d4651b14-773e-48a1-a041-0c64e9f8cb5f

Jain, J., \& Lyons, G. (2008). The gift of travel time. Journal of Transport Geography, 16, 8189. https://doi.org/10.1016/j.jtrangeo.2007.05.001

Kim, H. M., \& Kwan, M. P. (2013). Space-time accessibility measures: A geocomputational algorithm with a focus on the feasible opportunity set and possible activity duration. Journal of Geographical Systems, 5(1), 71-91. https://doi.org/10.1007/s101090300104

Lei, T. L., \& Church, R. L. (2010). Mapping transit-based access: integrating GIS, routes and schedules. International Journal of Geographical Information Science, 24(2), 283304. https://doi.org/10.1080/13658810902835404 
Li, Z., Zheng, J., \& Zhang, Y. (2019). Study on the Layout of 15-Minute Community-Life Circle in Third-Tier Cities Based on POI: Baoding City of Hebei Province. Engineering, 11, 592603. https://doi.org/10.4236/eng.2019.119041

Liu, S., \& Zhu, X. (2004). Accessibility analyst: an integrated GIS tool for accessibility analysis in urban transportation planning. Environment and Planning B: Planning and Design, 31(1), 105124. https://doi.org/10.1068/b305

López Lara, E. (1990): Una visión del papel territorial de la oferta de transporte público de pasajeros por carretera en la Comunidad Autónoma andaluza. Fundamentos para su reforma. Estudios Geográficos, 198, $65-$

81. http://estudiosgeograficos.revistas.csic.es/index.php/estudiosgeograficos/issue/archive

Manera, C., \& Garau, J. (2005). Los costes de la insularidad en el desarrollo económico. un contraste en el Mediterráneo occidental (1987-2002). In Colección Mediterráneo Económico (7) "Mediterráneo e Historia Económica". Alicante: Ed. Caja Rural Intermediterránea, Cajamar.

Martín Osante, J. M. (2020). Cohesión territorial y transporte aéreo en España, Cuadernos de Derecho Transnacional, 12(2), 527-545. https://doi.org/10.20318/cdt.2020.5620

Mavoa, S., Witten, K., McCreanor, T., \& O'Sullivan, D. (2012). GIS based destination accessibility via public transit and walking in Auckland, New Zealand. Journal of Transport Geography. 20(1), 15-22. https://doi.org/10.1016/j.jtrangeo.2011.10.001

Metra/Seis (1985). Análisis de viabilidad de una empresa de transporte aéreo de tercer nivel en Canarias. Las Palmas de Gran Canaria: Consejería de Turismo y Transportes del Gobierno de Canarias (Unpublished).

Minato, N., \& Morimoto R. (2011). Sustainable airline strategy using portfolio theory: A case study of remote islands in Japan. Journal of Air Transport Management, 17, 195198. https://doi.org/10.1016/j.jairtraman.2010.12.009

Ministerio de Fomento (2013). El transporte de viajeros entre Gran Canaria y Tenerife. Madrid: Secretaría General para los Servicios de Transportes ( $n^{\circ}$ 9.305).

Murillo Fort, C. (1992). Coste de la insularidad en Canarias. Las Palmas de Gran Canaria: Consejería de Economía y Hacienda del Gobierno de Canarias.

Neutens, T., Delafontaine, M., Schwanen, T., \& Van de Weghe, N. (2012). The relationship between opening hours and accessibility of public service delivery. Journal of Transport Geography, 25, 128-140. https://doi.org/10.1016/j.jtrangeo.2011.03.004 
Niemeier, D. A. (1997). Accessibility: an evaluation using consumer welfare. Transportation, 24(4), 377-396. https://doi.org/10.1023/A:1004914803019

Nutley, S. (1983). Transport policy apraisal and personal accesibility in rural Wales. Norwich: Geo Books.

Nutley, S. (1998). Rural areas: the accessibility problem. In B. Hoyle \& R. Knowles (Eds.), Modern Transport Geography (pp. 185-215). Chichester, Wiley.

Nutley, S. (2005). Monitoring rural travel behaviour: a longitudinal study in Northern Ireland 1979-2001. Journal of Transport Geography, 13, 247263. https://doi.org/10.1016/j.jtrangeo.2004.07.002

O'Sullivan, D., Morrison, A., \& Shearer, J. (2000). Using desktop GIS for the investigation of accessibility by public transport: an isochrone approach. International Journal of Geographical Information Science, 14, 85-104. https://doi.org/10.1080/136588100240976

Paci, R. (1997). Crescita economica e Sistemi Produttivi Locali in Sardegna. Cagliari: C.U.E.C.

Parras, M. A., \& Gómez, Érica L. (2015). Tiempo de viaje en transporte público. Aproximación conceptual y metodológica para su medición en la ciudad de Resistencia. Revista Transporte y Territorio, (13), 66-79. https://doi.org/10.34096/rtt.i13.1877

Pelfrene, E., Vlerick, P., Mak, R. P., De Smet, P., Kornitzer, M., \& De Backer, G. (2001). Scale reliability and validity of the Karasek 'Job Demand-Control-Support' model in the Belstress study. Work and Stress, 15(4), 297-313. https://doi.org/10.1080/02678370110086399

Polydoropoulou, A. \& Litinas, N. (2007). Chapter 9 Demand Models for Greek Passenger Shipping. Research in Transportation Economics, 21, 297322. https://doi.org/10.1016/S0739-8859(07)21009-1

Pooler, J. A. (1995). The use of spatial separation in the measurement of transportation accessibility. Transportation Research Part A: Policy and Practice, 29(6), 421427. https://doi.org/10.1016/0965-8564(95)00013-E

Price, L., \& Matthews, B. (2013). Travel time as quality time: parental attitudes to long distance travel with young children. Journal of Transport Geography, 32, 4955. https://doi.org/10.1016/j.jtrangeo.2013.08.001

Ramos Pérez, D. (2001). Transporte aéreo, territorio e insularidad en Canarias. Una aproximación en el umbral del siglo XXI. Santa Cruz de La Palma: Tauro Producciones. 
Ramos Pérez, D. (2015). Evaluación del impacto de la competencia en un mercado parcialmente protegido: El transporte aéreo interinsular en Canarias (2003-2012). Revista de Geografía Norte Grande, 61, 135-159. https://doi.org/10.4067/S0718-34022015000200008

Ramos Pérez, D. (2020). La bonificación de las tarifas aéreas a los residentes en los territorios no peninsulares de España (1993-2018). Boletín de la Asociación de Geógrafos Españoles, (86). https://doi.org/10.21138/bage.2942

Ranély Vergé-Dépré, C. (2005). Transport aérien et intégration territorial. L'exemple des Antilles françaises. Études caribéennes, 3. https://doi.org/10.4000/etudescaribeennes.563

Ray, I. (1985). Water transport versus air transport between $A$ \& $N$ Islands and mainland: an evaluation of social costs and benefits. Indian Journal of Regional Science, 17(1), 51-64.

Ritsema van Eck, J., Burghouwt, G., \& Dijst, M. (2005). Life styles, spatial configurations and quality of life in daily travel: an explorative simulation study. Journal of Transport Geography, 13(2), 123-134. https://doi.org/10.1016/j.jtrangeo.2004.04.013

Rigas, K. (2009). Boat or airplane? Passengers' perceptions of transport services to islands. The example of the Greek domestic leisure market. Journal of Transport Geography, 17(5), 396401. https://doi.org/10.1016/j.jtrangeo.2008.07.005

Rodrigue, J. P., Comtois, C., \& Slack B. (2006): The Geography of Transport Systems, London: Routledge.

Rodríguez, I. (1989). Accesibilidad de la población en el medio rural: el caso de San Martín de Valdeiglesias. Actas XI Congreso Nacional de Geografía, vol. I (pp. 206-211).

Rutz, W., \& Coull, J. (1996). Inter-island passenger shipping in Indonesia: development of the system: Present characteristics and future requirements. Journal of Transport Geography, 4(4), 275-286. https://doi.org/10.1016/S0966-6923(96)00028-2

Sambracos, E., \& Rigas, K. (2007). Passenger reactions to market deregulation: First results from the experience of the Greek islands market. Journal of Air Transport Management, 13(2), 6166. https://doi.org/10.1016/j.jairtraman.2006.11.007

Sjølander, J. (1999). Economic and social impact of smaller regional airports in Norway. Nairn: Forum on Air Transport in Europe's Remote Regions, Ed. Cranfield University and Luftfartsverket. Soo, J., Ettema, D., \& Ottens, H. F. L. (2009). Analysis of Travel Time in Multiple-Purpose Trips. Transportation Research Record, 2082, 56-62. https://doi.org/10.3141/2082-07 
Tahmasbia, B., \& Haghshenasab, H. (2019). Public transport accessibility measure based on weighted door to door travel time. Computers, Environment and Urban Systems, 76, 163177. https://doi.org/10.1016/j.compenvurbsys.2019.05.002

Thrift, N. y Pred, A. (1981). Time-geography: a new beginning. Progress in Human Geography, 5(2), 277-286. https://doi.org/10.1177/030913258100500209

Tsekeris, T. (2009). Dynamic analysis of air travel demand in competitive island markets. Journal of Air Transport Management, 267-

273. https://doi.org/10.1016/j.jairtraman.2008.11.008

Sri International (1992). Air transport study for the Canary Islands. Las Palmas de Gran Canaria: Consejería de Turismo y Transportes del Gobierno de Canarias (Unpublished).

UTE Eco-CoRe, (2019). Estudio sobre el coste privado de la ultraperiferia y la doble insularidad en Canarias. Las Palmas de Gran Canaria: Consejería de Economía y Hacienda del Gobierno de Canarias. Retrieved from http://www.datosdelanzarote.com/Uploads/doc/Estudio-sobre-elcoste-privado-de-la-ultraperiferia-y-la-doble-insularidad-en-Canarias-20190625100430600Estudiosobrecostes-definitivo.pdf

Wardman, M. (1998). The value of travel time: A review of British evidence. Journal of Transport Economics and Policy, 32(3), 285-316. https://www.jstor.org/stable/20053775

Williams, G. (1999). Dependency of Europe's remoter regions on air transport. Forum on Air Transport in Europe's Remote Regions. Nairn: Cranfield University y Lufffartsverket.

Yamaguchi, K. (2007). Inter-regional air transport accessibility and macro-economic performance in Japan. Transportation Research Part E: Logistics and Transportation Review, 43(3), 247-258. https://doi.org/10.1016/j.tre.2006.10.004

Yatskiv, I. (2017). Accessibility to Riga public transport services for transit passengers. Procedia Engineering, 187, 82-88. https://doi.org/10.1016/j.proeng.2017.04.353 